OPEN ACCESS

Edited by:

Musthafa Mohamed Essa,

Sultan Qaboos University, Oman

Reviewed by:

Samir Al-Adawi,

Department of Behavioral Medicine

College of Medicine and Health

Science, Sultan Qaboos University,

Oman

Judie Arulappan

College of Nursing, Sultan Qaboos

University, Oman

Ye Xiong,

Henry Ford Health System,

United States

*Correspondence:

Aurélie Ledreux

Aurelie.Ledreux@du.edu

Specialty section:

This article was submitted to

Neurodegeneration,

a section of the journal

Frontiers in Neuroscience

Received: 24 April 2020

Accepted: 29 June 2020

Published: 30 July 2020

Citation:

Ledreux A, Pryhoda MK,

Gorgens K, Shelburne K, Gilmore A, Linseman DA, Fleming H, Koza LA,

Campbell J, Wolff A, Kelly JP,

Margittai $M$, Davidson BS and

Granholm A-C (2020) Assessment

of Long-Term Effects

of Sports-Related Concussions:

Biological Mechanisms and Exosomal Biomarkers. Front. Neurosci. 14:761.

doi: 10.3389/fnins.2020.00761

\section{Assessment of Long-Term Effects of Sports-Related Concussions: Biological Mechanisms and Exosomal Biomarkers}

\author{
Aurélie Ledreux ${ }^{1 *}$, Moira K. Pryhoda ${ }^{2}, K_{i m}$ Gorgens ${ }^{3}$, Kevin Shelburne', Anah Gilmore', \\ Daniel A. Linseman ${ }^{1,4}$, Holly Fleming ${ }^{4}$, Lilia A. Koza ${ }^{1,4}$, Julie Campbell ${ }^{5}$, Adam Wolff6, \\ James P. Kelly ${ }^{7}$, Martin Margittai ${ }^{8}$, Bradley S. Davidson² and Ann-Charlotte Granholm ${ }^{1}$ \\ ${ }^{1}$ Knoebel Institute for Healthy Aging, University of Denver, Denver, CO, United States, ${ }^{2}$ Department of Mechanical \\ and Materials Engineering, University of Denver, Denver, CO, United States, ${ }^{3}$ Graduate School of Professional Psychology, \\ University of Denver, Denver, CO, United States, ${ }^{4}$ Biological Sciences, University of Denver, Denver, CO, United States, \\ ${ }^{5}$ Pioneer Health and Performance, University of Denver, Denver, CO, United States, ${ }^{6}$ Denver Neurological Clinic, Denver, \\ CO, United States, ${ }^{7}$ Marcus Institute for Brain Health, Department of Neurology, University of Colorado Anschutz Medical \\ Campus, Aurora, CO, United States, ${ }^{8}$ Department of Chemistry and Biochemistry, University of Denver, Denver, CO, \\ United States
}

Concussion or mild traumatic brain injury (mTBI) in athletes can cause persistent symptoms, known as post-concussion syndrome (PCS), and repeated injuries may increase the long-term risk for an athlete to develop neurodegenerative diseases such as chronic traumatic encephalopathy (CTE), and Alzheimer's disease (AD). The Center for Disease Control estimates that up to 3.8 million sport-related mTBI are reported each year in the United States. Despite the magnitude of the phenomenon, there is a current lack of comprehensive prognostic indicators and research has shown that available monitoring tools are moderately sensitive to short-term concussion effects but less sensitive to long-term consequences. The overall aim of this review is to discuss novel, quantitative, and objective measurements that can predict long-term outcomes following repeated sports-related mTBls. The specific objectives were (1) to provide an overview of the current clinical and biomechanical tools available to health practitioners to ensure recovery after mTBls, (2) to synthesize potential biological mechanisms in animal models underlying the long-term adverse consequences of mTBls, (3) to discuss the possible link between repeated mTBI and neurodegenerative diseases, and (4) to discuss the current knowledge about fluid biomarkers for mTBls with a focus on novel exosomal biomarkers. The conclusions from this review are that current post-concussion clinical tests are not sufficiently sensitive to injury and do not accurately quantify post-concussion alterations associated with repeated mTBls. In the current review, it is proposed that current practices should be amended to include a repeated symptom inventory, a cognitive assessment of executive function and impulse control, an instrumented assessment of balance, vestibulo-ocular assessments, and an improved panel of blood or exosome biomarkers.

Keywords: concussion, mild traumatic brain injury, biomechanics, blood biomarkers, exosomes, neuropathology, neurodegenerative disease risk 


\section{INTRODUCTION}

Up to 3.8 million sports-related concussions (mild traumatic brain injury; hereafter referred to as mTBI) are diagnosed annually in the United States; however, it is estimated that only $50 \%$ of mTBIs are reported (Broglio et al., 2017; Iverson et al., 2017). A potential consequence of mTBI is post-concussion syndrome (PCS), defined as the occurrence of clinical symptoms that continue for weeks, or months following the incident (Sosnoff et al., 2011; Berz et al., 2013; Tagge et al., 2018). Related PCS physical symptoms can include headache, dizziness, insomnia, exercise intolerance, fatigue, as well as noise, and light sensitivity (Ling et al., 2015; Cottle et al., 2017; Iverson et al., 2017; Gozt et al., 2020). Psychological symptoms including depression, irritability, and anxiety are common. Cognitive problems such as memory loss, poor concentration, and reduced problem-solving skills can also persist over time (Mclinnes et al., 2017; Howell et al., 2018a). These long-lasting physical, psychological, and cognitive symptoms can significantly affect quality of life, prevent return to work and add a financial burden on individuals experiencing PCS (Voormolen et al., 2019). Studies have identified a number of risk factors including age, sex, learning issues, history of migraine that could influence development of PCS (Iverson et al., 2017). Both acute and chronic PCS have been identified in the literature, each presenting with unique health concerns (Tagge et al., 2018; Yin et al., 2019; Scott et al., 2020). In addition, there is increased concern that repeated mTBIs may contribute to adverse long-term consequences including neurodegenerative diseases (Asken et al., 2016; McAllister and McCrea, 2017). It is thought that one or several mTBIs give rise to alterations in inflammatory processes in the brain via activation of astrocytes and microglial cells (Mouzon et al., 2014; Papa et al., 2015; Winston et al., 2016), as well as the more direct neuronal insults including axonal degeneration, neuronal cell loss (Daneshvar et al., 2015; McKee and Daneshvar, 2015), and intracranial accumulation of dementia-related damaging proteins including amyloid beta peptide $(A \beta)$, and phosphorylated forms of Tau ( $p$-Tau; Papa et al., 2015; Gill et al., 2018). In a recent study, our group demonstrated that neuron-derived exosomal (NDE) biomarkers, isolated from blood samples, exhibit significant alterations both short- and long-term following one or repeated mTBIs (Goetzl et al., 2019a). Although research on the short- and long-term effects of mTBI have been conducted in animal models (see below), reliable and reproducible assessments for clinical use including biomarkers are still under development in this field. The development of biomarker panels is important since they may predict or quantify long-term health risks for adverse outcomes and may represent a more objective measure of the effectiveness of interventions. Development of novel assessment tools is needed to better quantify injury in the short-term and to fully understand the long-term consequences of mTBIs for brain health.

Demographic studies have identified several risk factors for sustaining sports-related mTBIs. The greatest risk of sustaining an mTBI exists among males playing American football or Australian rugby, females playing soccer (Giza et al., 2013;
Kutcher and Giza, 2014), and both genders playing ice hockey (Simmons et al., 2017). Athletes participating in lacrosse were recently shown to have a high risk for mTBIs, although fewer studies have been conducted in terms of injury consequences in lacrosse compared to other high impact sports (Reynolds et al., 2016; Miyashita et al., 2017; O'connor et al., 2017). Athletes playing baseball, softball, volleyball, and gymnastics reportedly have a lower risk for sustaining mTBIs compared to other contact sports (Giza et al., 2013). Across all sports, females, and younger players were reported to have an increased risk of developing PCS after sustaining one or repeated mTBIs (Giza et al., 2013; Collins et al., 2014; Kutcher and Giza, 2014). A personal history of migraine, depression, previous concussions, learning disabilities, or attention-deficit hyperactivity disorder (ADHD) also increases the risk for developing PCS following mTBI (Giza et al., 2013; Bramley et al., 2016). Thus, several different factors impact the risk for long-term consequences after mTBI, including the severity of the injury, the type of sports and position played, age of the injured person (younger individuals suffer fewer longterm consequences), number of previous mTBIs, and the addition of repeated subclinical, "sub-concussive" injuries (Asken et al., 2016). The demographics of our cohort of National Collegiate Athletic Association (NCAA) Division 1 athletes resembles the demographics described above, in terms of increased risks sustained in athletes playing ice hockey, or lacrosse (see Table 1). An increase in concussions in skiers (44\% with a history of concussions), similar to previously reported findings (Gil et al., 2017) was also observed.

Research on the typical neurocognitive sequelae of concussions suggests there is little uniformity in the presentation and course of PCS (Leddy et al., 2016). Despite that, the diagnosis of concussion is commonly based on physical, cognitive, and emotional symptoms, including the presence or absence of loss of consciousness and/or amnesia, behavioral changes, and/or sleep disturbance (Kutcher and Giza, 2014; Broglio et al., 2017; Cottle et al., 2017; Iverson et al., 2017). The NCAA adopted its Concussion Policy and Legislation in 2010 (Baugh et al., 2015), which recommends that all athletes participate in a brain injury/concussion history survey, symptom evaluation, cognitive assessment, and balance evaluation prior to participation in a collegiate sport but these measures of cognitive change, balance, and self-reported distress are only moderately sensitive to mTBI (Higgins et al., 2017). Further, despite these guidelines, many competitive athletes underreport post-concussive symptoms and fail to report concussive injuries (Covassin et al., 2007; Kroshus et al., 2015; McDonald et al., 2016; Wallace et al., 2017). As a result, they do not receive the clinical attention needed to promote recovery and avoid short- and long-term risks.

The accurate assessment of mTBI and post-concussive symptoms is crucial to athlete safety and is often at the center of return-to-play (RTP) decisions for healthcare providers and sports medicine staff (Baugh et al., 2015; Broglio et al., 2017). Importantly, premature cognitive and physical activity prior to the recovery of concussive injury is associated with prolonged neuronal vulnerability, often increasing the severity of and delaying the resolution of PCS (Harmon et al., 2013). Additionally, individuals who sustain one or several additional 
TABLE 1 | History of concussion in cohort by sport and gender.

\begin{tabular}{|c|c|c|c|c|}
\hline Sport & $\begin{array}{c}\text { Total number of } \\
\text { athletes }\end{array}$ & $\begin{array}{l}\text { Number of athletes with } \\
\text { history of concussion }\end{array}$ & $\begin{array}{l}\text { Percent of athletes with } \\
\text { history of concussion }\end{array}$ & $\begin{array}{c}\text { Male/Female with } \\
\text { history of concussion }\end{array}$ \\
\hline Basketball & 34 & 14 & $41 \%$ & $8 / 6$ \\
\hline Diving & 6 & 3 & $50 \%$ & $2 / 1$ \\
\hline Golf & 10 & 0 & $0 \%$ & $0 / 0$ \\
\hline Gymnastics & 15 & 4 & $27 \%$ & $0 / 4$ \\
\hline Hockey & 43 & 21 & $49 \%$ & $21 / 0$ \\
\hline Lacrosse & 116 & 46 & $40 \%$ & $31 / 15$ \\
\hline Skiing & 18 & 8 & $44 \%$ & $3 / 5$ \\
\hline Soccer & 48 & 21 & $44 \%$ & $9 / 12$ \\
\hline Swimming & 27 & 4 & $15 \%$ & $3 / 1$ \\
\hline Tennis & 20 & 3 & $15 \%$ & $3 / 0$ \\
\hline Volleyball & 16 & 4 & $25 \%$ & 0/4 \\
\hline Total & 353 & 128 & $36 \%$ & $80 / 48$ \\
\hline
\end{tabular}

concussions may be more vulnerable to a worsening of post-concussive neurological and metabolic dysfunction and potentially to long-term harm to the brain (Prins et al., 2010; Kenney et al., 2018). Despite the increased attention to PCS and the risk of long-term negative effects on brain health, few quantitative methods are available to fully assess these risks. There is an urgent need to improve TBI diagnosis and support recovery in the short-term and to understand and reduce the long-term risk of adverse outcomes including neurodegenerative conditions such as chronic traumatic encephalopathy (CTE) or Alzheimer's disease $(\mathrm{AD})$ that are often associated with repeated concussive events.

The objective of this review is to provide an overview of: (1) clinical and biomechanical tools available to sport medicine practitioners to ensure recovery after mTBIs before RTP, (2) potential biological mechanisms in animal models underlying long-term adverse consequences of mTBIs, (3) the link between repeated $\mathrm{mTBI}$ and neurodegenerative diseases, and (4) current knowledge of fluid biomarkers for mTBIs, with an emphasis on blood biomarkers and on emerging exosomal biomarkers. More research into specific mechanisms of injury is needed to improve the prognostics for sport-related mTBIs and a better understanding of the neuronal consequences reflected by plasma or exosome biomarkers is also necessary.

\section{CURRENT ASSESSMENT TOOLS FOR MTBI}

General guidance for the management of sports-related mTBIs includes an assessment of symptoms and disposition after first addressing acute trauma (McCrory et al., 2017). Injury management guidelines and many state statutes require that concussed players not be allowed to return to play on the day of the injury (Harmon et al., 2013; McCrory et al., 2017). A graduated six-step RTP protocol after a concussion is suggested by the Consensus Statement on Concussion in Sport (McCrory et al., 2017; Patricios et al., 2018).

The diagnosis of concussion has historically been based on self-reported symptoms (Kasamatsu et al., 2016) including nausea, dizziness, or headache. Six core symptom scales have been developed for sport-related concussion (Alla et al., 2011; Thomas et al., 2011) and many of them are variants of the original Pittsburgh Steelers Post-Concussion Scale (Alla et al., 2011; McLeod and Leach, 2012). These scales were developed from clinical experience and consensus rather than empirical evidence (Cantu, 1997; Leclerc et al., 2001; Tagge et al., 2018).

The Concussion in Sport Group (CISG) first introduced the Sport Concussion Assessment Tool (SCAT) 1 to provide a multifaceted standardized assessment of concussion which has since evolved into the SCAT2, SCAT3, and SCAT5 (McLeod and Leach, 2012; Yengo-Kahn et al., 2016; Echemendia et al., 2017; Joseph et al., 2018). The diagnostic utility of the SCAT appears to diminish 3-5 days post-injury (Yengo-Kahn et al., 2016), making this test less effective for evaluating post-acute effects including PCS. Additionally, consensus statements from the CISG state that grading systems should not be used to dictate concussion management, and that the SCAT5 symptom checklist does not demonstrate clinical utility in tracking recovery (McCrory et al., 2017). Despite that, $87 \%$ of athletic trainers report that they will return a non-symptomatic athlete to play based only on selfreported symptoms (Covassin et al., 2009). This may be especially problematic in collegiate or professional sports where athletes have a financial incentive to play. For those reasons, self-reported symptom scores should always be used in tandem with objectively measured domains during concussion management. Given the heterogeneity of mTBI presentation and the high stakes of athlete health, there is an ongoing need to develop more sensitive instruments and individualized injury management protocols (Choe and Giza, 2015).

Because cognitive dysfunction has been demonstrated to take longer to resolve than the self-reported symptoms (Carman et al., 2015), computerized neurocognitive tests have become the most commonly used objective clinical measure of mTBI. The Immediate Post-Concussion Assessment and Cognitive Test (ImPACT; Kontos et al., 2014; Higgins et al., 2017) is widely used in both youth and NCAA sports for concussion management in the United States. ImPACT was found to have $81.9 \%$ sensitivity and $89.4 \%$ specificity to mTBI (Schatz et al., 2006) and generates composite scores for four domains: 
Verbal Memory, Visual Memory, Processing Speed, and Reaction Time, of which visual memory, and reaction time are the most sensitive domains to the cognitive changes following concussion (Covassin et al., 2007; Majerske et al., 2008). Other computerized neurocognitive assessment tools such as the ANAM $^{\text {TM }}$ Core Battery (Kane et al., 2007) can measure changes in attention, working memory, and cognitive efficiency, and may therefore be even more sensitive to the long-term effects of mTBI on brain health (Covassin et al., 2007; Kane et al., 2007; Majerske et al., 2008; Kontos et al., 2014; Martini et al., 2017). Preliminary data from our cohort (Gorgens et al., unpublished observations) strongly suggest that executive function, reaction time, and processing speed should be included in the routine assessment of PCS, since these cognitive changes appear to linger after most other cognitive functions have returned to baseline.

Vestibular- and ocular-based clinical tests are also sensitive to concussive injury. The vestibular and ocular systems contribute critical information on spatial orientation to the central nervous system (CNS) and detriments in the vestibulo-ocular system can lead to dizziness, nausea, and balance impairment. In a battery of vestibular-based laboratory tests, post-concussion deficits can be identified using the Dynamic Visual Acuity Test (DVAT; Zhou and Brodsky, 2015), and the King-Devick (KD) test (King et al., 2015). The KD test measures timed saccadic eye movements and has been shown to have $86 \%$ sensitivity and $90 \%$ specificity to concussion (Galetta et al., 2011), and is also sensitive to unreported mTBIs (King et al., 2015). The addition of the KD test may increase the overall sensitivity of a clinical concussion battery.

The call for more sensitive concussion assessment and the growing awareness of CNS compensatory function has increased the utility of balance testing. Compensatory CNS function is observed when, for example, an "eyes closed" balance task results in instability. In that case, vision can be said to compensate for deficits in the vestibular system (Sosnoff et al., 2011). In fact, the visual system has been determined by sensory organization testing to be dominant over the vestibular system in balance tasks (Liaw et al., 2009; Allum and Honegger, 2016). An mTBI results in a deficit of vestibular function and so, when balancing, concussed patients will compensate for this loss using the visual system.

The Balance Error Scoring System (BESS; Finnoff et al., 2009; Yengo-Kahn et al., 2016; Miyashita et al., 2017) is an observational diagnostic tool frequently used to test static balance post-mTBI on firm and foam surfaces with the eyes closed. The BESS is the most commonly used balance metric for sideline concussion diagnosis despite concerns that it does not adequately assess vestibular function (Kalajainen, 2015) and issues about learning effects with repeated testing (Valovich et al., 2004; Mulligan et al., 2013). However, the BESS is used in most NCAA protocols for mTBIs, as this assessment is readily available in the field. Instrumented measurements of static balance have been found to be less subject to scoring errors than observational balance tools and more sensitive to mTBI-related changes in balance. For example, concussed football players showed greater center of pressure displacement immediately after injury with an improvement of function before RTP (Powers et al., 2014).
Measuring dynamic balance (balance during movement) may be a better indicator of athlete readiness to RTP. However, clinicians are currently limited in their ability to measure dynamic balance as those methods are still being optimized. A slower normal gait speed (Howell et al., 2018a) has been documented among patients with mTBIs. Tandem gait, defined as walking toe to heel, is also slower for the first three days after concussion, and dual task tandem gait time is slower for the first two weeks post-mTBI (Howell et al., 2018a). Other measurements such as smaller step width (Buckley et al., 2019) and slower dual task cadence (Howell et al., 2018b) have been documented post-mTBI. Gait disturbance has also been found in neurodegenerative disease and aging, which may give insight into longer-term effects of multiple mTBIs. For example, the walking speed of patients with $\mathrm{AD}$ is slower than healthy older adults (Johansson et al., 2017) and walking speed differences appear already in those with mild cognitive impairment (MCI; Knapstad et al., 2019), suggesting that gait change is an early event and can be used to diagnose potential problems. It is therefore possible that long-term tracking of gait post-mTBI can reveal future risk for developing neurodegenerative conditions related to multiple mTBIs and may represent a sensitive dynamic balance measure for these cases.

Chen and Chou (2010) have created a clinically-accessible dynamic biomechanical balance measure using reflective marker data from the ankle to create a Center of Mass (COM)-ankle inclination measure. COM medio-lateral displacement is typical of concussed patients in the first two days post-concussion (Grants et al., 2017) and up to 2 months post-concussion (Howell et al., 2018a). Peak anterior COM velocity can also be measured and is observed to be greater for the first two months postconcussion (Howell et al., 2018a). There is continued work on the development of a commercially-viable quantitative measurement of dynamic balance. The use of the Kinect system (Microsoft, United States) for spatiotemporal parameters of gait has been promising (Zhu et al., 2017).

In sum, there is a growing base of concussion research that confirms our understanding of concussion as a multi-faceted injury, affecting both cognitive, and balance systems. To date, the measurement of mTBI-related balance and cognitive sequelae as required by NCAA regulations is moderately sensitive to shortterm concussion effects, but less sensitive for long-term PCS.

\section{POTENTIAL BIOLOGICAL MECHANISMS - ANIMAL STUDIES}

Most biological mechanisms for long-term effects of mTBIs have been discovered via animal models. There have been several thorough reviews published previously describing the relative pros and cons of various mouse and rat models of TBI (Lyeth, 2016; Osier and Dixon, 2016; Wojnarowicz et al., 2017). Therefore, only a brief overview is provided here. There are many distinct methods for inducing TBI in rodent models for research purposes. A partial listing of the many variants of these models is provided by Xiong et al. (2017a). However, in terms of mTBI or concussion, it could be argued that the most physiologically 
relevant models are those that utilize pneumatically-driven or electromagnetic impact devices to deliver single or repetitive closed head injuries to mice or rats. Recent studies have begun to use un-anesthetized animals and animals in which the head is free to move in response to the impact rather than being immobilized. These models may ultimately prove to replicate the dynamic injury of mTBI more accurately than those using anesthetized and immobilized animals; however, they are not without some level of controversy.

\section{Diffuse Axonal Injury}

Several models of repetitive mTBIs have demonstrated persistent multi-focal axonal injury using electron microscopy, silver staining, and diffusion tensor imaging (Shitaka et al., 2011; Bennett et al., 2012). Axonal injury was prevalent in repetitive mTBI models 3 to 6 months post-injury (Ojo et al., 2016). Moreover, in comparison to the axonal injury observed following a single mTBI, repetitive mTBIs exacerbated axonal degeneration and this degenerative process was ongoing in subcortical white matter tracts for up to 24 months post-injury, suggesting long-term damage (Laurer et al., 2001; Mouzon et al., 2012, 2014, 2018). Thus, repetitive mTBIs can induce chronic and progressive axonal degeneration which undoubtedly contributes to cognitive and perhaps emotional deficits post-mTBI. It seems likely that this ongoing degenerative process may set the stage for the development of chronic neurodegenerative disorders, even though definitive proof of this link has not yet been provided in humans.

\section{Tau Pathology}

Although neither of the specific Tau pathologies of AD or CTE have been precisely replicated in mouse models of mTBI, marked alterations in Tau phosphorylation, and processing do occur in mice following TBIs. For instance, a single unilateral impact in un-anesthetized mice induced rapid tauopathy within $24 \mathrm{~h}$ of injury, which persisted and progressed to the contralateral uninjured cortex by 5.5 months post-injury (Tagge et al., 2018). Several other models of single or repetitive mTBIs also produce aberrant Tau pathology (Kane et al., 2012; Luo et al., 2014; Petraglia et al., 2014; Yang et al., 2015; Ignowski et al., 2018). Of note, un-anesthetized mice administered 6 concussive impacts daily for 7 days, displayed elevated cortical p-Tau immunoreactivity up to 6 months post-mTBI (Petraglia et al., 2014). Collectively, these results demonstrate that increased p-Tau is observed in diverse mouse models of mTBI. Moreover, this phospho-Tau pathology often persists for several months post-injury and has the potential to "spread" to areas of the brain which are outside of the primary injury site via a seeding/prionlike mechanism, perhaps via exosomes (Deleo and Ikezu, 2018; Winston et al., 2019a). Therefore, these mouse studies have been essential for informing the biomarker field as to which biomarkers of disease may be of the most relevance to long-term pathology in the brain.

The effects of repetitive mTBIs have been investigated in two mouse models of tauopathy. In transgenic mice expressing the shortest human Tau isoform (hTau23), repetitive mTBI induced extensive telencephalic neurofibrillary tangles (NFTs) in only one of twelve transgenic mice (Yoshiyama et al., 2005). In a second model, aged (18-month-old) mice, which express multiple wildtype human Tau isoforms (hTau mice), were subjected to either a single or repeated mTBIs. Although these aged hTau mice already express significant Tau pathology, only repetitive mTBI markedly increased hyper-phosphorylation of Tau as assessed by multiple phospho-Tau specific antibodies. Thus, it appears that repetitive mTBIs have the potential to exacerbate certain forms of Tau pathology, although precipitation of bona fide NFTs is not efficiently induced by this trauma/gene interaction paradigm, at least not in mouse models. To our knowledge, only one laboratory has described a mouse model which seems to meet (most of) these criteria. This model induces a tauopathy marked by hyperphosphorylation which was detectable in cortical axons ipsilateral to a cortical impact injury by $24 \mathrm{~h}$ post-injury and in neuronal soma by 2 weeks post-injury (Tagge et al., 2018). Moreover, the Tau pathology "spread" to the distant cortex in a bilateral fashion at 5.5 months post-injury. Finally, these authors have also demonstrated that cis phospho-Tau is a proximal driver of neuronal injury post-TBI and that treatment with a specific cis phospho-Tau blocking antibody significantly attenuates Tau pathology and spread (Albayram et al., 2017), providing potential new avenues for development of effective preventative methods after repeated mTBIs in humans.

\section{Neuroinflammation}

It is well known that recovery from neurotrauma, such as a stroke or TBI, can be confounded by the brain's ability to perpetually activate microglia. Reactive gliosis following neural injury can lead to chronic inflammation and neurodegeneration (Turner et al., 2016). Undoubtedly, neuroinflammation is the most common pathological finding in diverse mouse models of mTBI and is an undeniable contributing factor in chronic neurodegenerative disease states (Mouzon et al., 2014; Daneshvar et al., 2015; McAteer et al., 2016; Winston et al., 2016; Edwards et al., 2017). The activation of astrocytes in response to neuronal damage, i.e., astrogliosis, has been identified as an early event following a single mTBI in juvenile mice subjected to closed head injury, adult mice exposed to controlled cortical impact, and adult un-anesthetized mice given a lateral closed head impact injury (Susarla et al., 2014; Israel et al., 2016; Chen et al., 2017; Rodriguez-Grande et al., 2018; Tagge et al., 2018). In some cases, astrogliosis persisted for up to 28 days post-TBI, which was the longest time point analyzed post-injury in these studies (Susarla et al., 2014). Our group has similarly observed astrogliosis in mice exposed to mTBI induced by controlled cortical impact (see Figure 1; Ignowski et al., 2018). In mice subjected to repeated mTBIs, long-term cognitive deficits were associated with persistent astrogliosis up to one-year post-injury (Mannix et al., 2013). Crawford and colleagues have examined the lifelong consequences of repetitive mTBIs in mice and found that neuroinflammation progressed over 12 to 24 months, appearing in subcortical white matter tracts in association with degenerating axons at these later time points post-injury (Mouzon et al., 2012, 2014, 2018). These findings suggest that repetitive mTBIs can precipitate a lifelong neurodegenerative process. Neuroinflammation has been observed as a contributing 

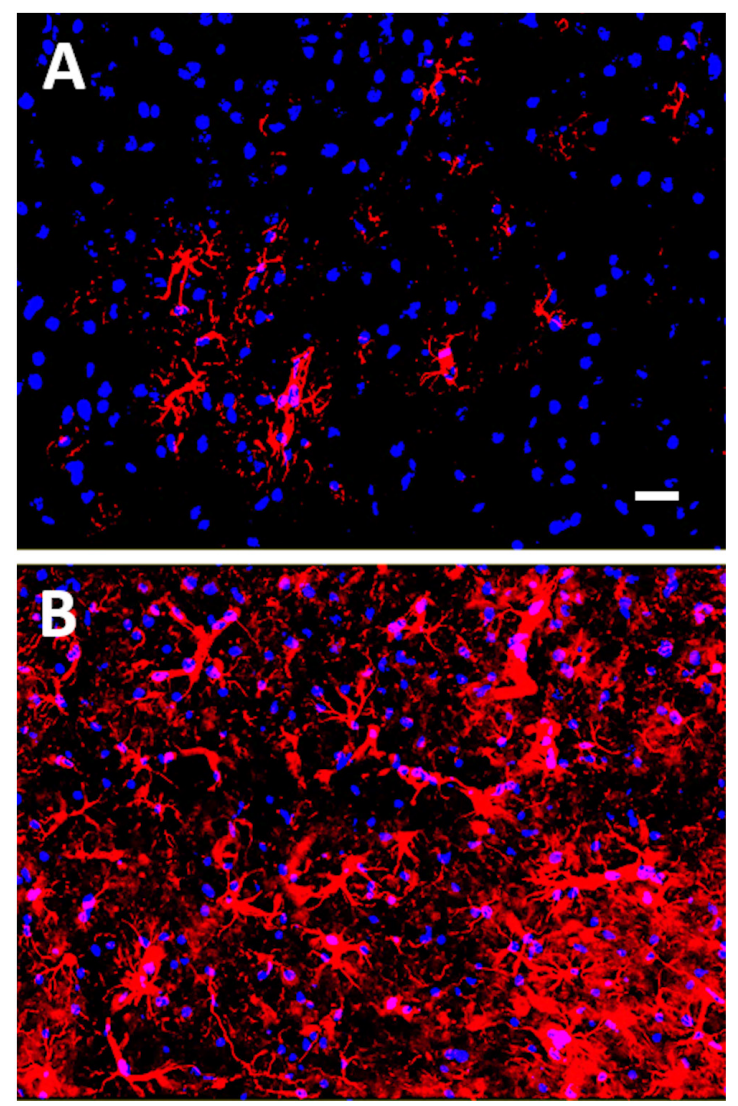

FIGURE 1 | Glial fibrillary acidic protein (GFAP) immunostaining of mouse cortex 18 days post-mTBI induced by a closed head injury using an electromagnetically controlled impact device. In (A), cortex of a Sham mouse, and in (B), cortex of a mouse subjected to mTBI. GFAP-positive astrocytes are shown in red (Сy3) and nuclei are shown in blue (Hoechst dye). Note the increased gliosis in mouse cortex following the mTBI. Scale bar represents 20 microns.

factor, both in CTE (Daneshvar et al., 2015; McAteer et al., 2016; Tagge et al., 2018), and in AD (Heneka et al., 2015).

\section{Alzheimer's Disease (AD)}

In two distinct models of $\mathrm{A} \beta$ precursor protein (APP) mutagenesis, the Tg2576 mouse and the APP/PS1 transgenic mouse, mTBI enhanced $A \beta$ aggregation, and precipitated cognitive impairment of pre-symptomatic AD mice (Uryu et al., 2002; Tajiri et al., 2013). In contrast, repetitive mTBI did not increase $A \beta$ or phospho-Tau levels in the 3xTg-AD mouse model. However, axonal degeneration and microglial activation were observed in these animals up to 1 year post-TBI (Winston et al., 2016). Thus, consistent findings from several groups unequivocally suggest that neurotrauma does at the very least accelerate the development of $\mathrm{AD}$ changes in genetically predisposed rodents. Overall, these findings are somewhat variable, but they suggest that mTBI administered on a genetic background of human genes expressing $\mathrm{AD}$ pathology tends to accelerate amyloid pathology, hippocampal neurodegeneration, and neuroinflammation. Additional studies are underway in our group to further investigate the effects of mTBI on the progression of $\mathrm{AD}$ neuropathology.

In conclusion, these previous studies suggest that a genetic propensity for AD pathology is exacerbated by repeated mTBIs, thus providing a potential biological mechanism for the fact that not everyone with multiple mTBIs, either resulting from active duty or from high impact sports, succumb to neurodegenerative diseases including CTE when they get older. Animal studies are valuable in that they can dissect long-term effects in a relatively short time period, and researchers can modify specific genetic factors to explore their impact on long-term brain health.

\section{CONCUSSION AND NEURODEGENERATIVE DISEASES}

Although several sources conclude no connection between exposure to a single mTBI and incidence of neurodegenerative disease (Guskiewicz et al., 2005; Carson, 2017; Manley et al., 2017; McCrory et al., 2017; Lobue et al., 2020), other studies suggest that repeated mTBIs, without full recovery time between events, could increase the risk for neurodegenerative disease decades later (Guskiewicz et al., 2005; Cruz-Haces et al., 2017). Repetitive injuries are known to increase the risk for future injury and are associated with slower recovery after an mTBI including the persistence of PCS (Daneshvar et al., 2011). Guskiewicz et al. (2005) documented earlier than typical onset of AD in a cohort of retired American football players. In that study, the football players who reported three or more mTBIs had five times greater MCI diagnoses and three times greater reported memory problems compared to players who did not report a history of mTBIs. CTE is a complex neurological disorder characterized by executive dysfunction, depression, memory impairment, and dementia, amongst other types of cognitive and affective dysfunctions (Daneshvar et al., 2015; Asken et al., 2016; McKee et al., 2016; Armstrong et al., 2017). CTE has been strongly associated with one major TBI or repeated milder mTBIs.

In a recent post mortem study, investigators found evidence of CTE in the brains of teenage athletes following repeated mTBIs (Tagge et al., 2018), suggesting that the onset of pathology may be earlier than previously thought. The neuropathological alterations that occur following repeated brain injuries include diffuse axonal damage (Lepage et al., 2018), white matter degeneration, widespread neuroinflammation with activated microglial cells, and astrocytes (Chen et al., 2017; Collins-Praino and Corrigan, 2017; Johnson et al., 2017), lipid peroxidation and other oxidative damage (Yates et al., 2017), as well as accumulation of intracellular NFTs caused by p-Tau (Olczak et al., 2017). The accumulation of NFTs has been observed in the deep sulci in frontal and temporal cortices and along cortical blood vessels of individuals with CTE and is thought to contribute significantly to the long-term development of neurodegeneration in individuals with mTBIs (Omalu, 2014; McKee et al., 2016; Washington et al., 2016; Mez et al., 2017; Alosco et al., 2018). Dizziness and imbalance, both common symptoms of mTBI, have been linked to white matter abnormalities and diffuse axonal injuries (Cecil et al., 1998), in addition to vestibular system 
damage (Akin et al., 2017). Diffuse axonal injuries following mTBIs have also been reported in experimental animal models of TBI (Mannix et al., 2013).

Tau pathology induced by mTBI may be related to both $\mathrm{AD}$ and CTE, which each have unique presentations of $\mathrm{p}$-Tau species. In $\mathrm{AD}$, Tau is hyper-phosphorylated and aggregated into NFTs that are found throughout the hippocampus and cortex and exhibit a progressive spread eventually reaching all cortical areas (Raj et al., 2012; Kaufman et al., 2016; Furman et al., 2017). In contrast, the Tau pathology in CTE is characterized by the accumulation of abnormal (and hyperphosphorylated) Tau focally around small blood vessels at the depths of the sulci within the cortex, essentially at the white matter-gray matter interface (Omalu, 2014; McKee et al., 2016; Mez et al., 2017; Olczak et al., 2017; Tagge et al., 2018). Notably, Tau filaments in $\mathrm{AD}$ and CTE assume different molecular structures (Fitzpatrick et al., 2017; Falcon et al., 2019). In a recent study by Mez et al. (2017), the investigators examined the brains of 202 former American football players who had passed away. CTE was diagnosed in 177 of these players, with the mildest pathology observed in athletes who had only played football in high school, and the most severe pathology in players who had a professional football career (Mez et al., 2017). Eighty-six percent of the athletes who played professionally exhibited severe CTErelated neuropathology. Among the participants with severe CTE pathology, $85 \%$ showed clinical signs of dementia. Even though this was a select collection of brain tissue obtained because the athletes actively donated their brains to this particular project, these findings are alarming and suggest that sustaining repeated mTBIs is related to significant detriments later in life. These pathology studies also suggest that total Tau and p-Tau need to be included in any blood biomarker panel that is used to assess longterm mTBI effects, and that repeated mTBIs earlier in life most certainly predisposes individuals to neuropathology later in life.

Mild traumatic brain injurys may also trigger other neurodegenerative diseases including Parkinson's disease (PD) and amyotrophic lateral sclerosis (ALS; Gupta and Sen, 2016; Gardner et al., 2018), although there are less quantifiable data available to firmly connect mTBIs with the incidence of either PD or ALS to date. The strong connection between $\mathrm{p}$-Tau and CTE as well as AD has prompted a wealth of investigations to reveal blood or cerebrospinal fluid (CSF) biomarkers related to toxic forms of Tau both acutely and chronically after mTBIs. TBI and PCS share many clinical symptoms with CTE and may even precede the development of CTE. Although most biomarker studies to date have been focused on short-term (weeks or months) consequences of mTBIs, one should also take into consideration the long-term effects (years or decades) of repeated mTBIs that take place in youth or collegiate high-impact sports.

\section{BIOMARKERS IN mTBI}

\section{Blood Biomarkers}

The relationship between multiple mTBIs and neurodegenerative diseases is a controversial issue that is currently being debated in several lawsuits. Because of the inherent difficulty in providing tangible evidence of long-term risk after multiple mTBIs in professional players, prognostic biomarkers are needed to provide confirmation of damage and association with neurodegenerative conditions. Fluid biomarker research has gained increasing attention over the last decade for its promise to help quantify injury severity, determine when it is safe for athletes to return to play, and to track the long-term consequences of mTBIs for brain health. That research also highlights the underlying biological mechanisms of mTBI and can be used to evaluate the effectiveness of therapeutic interventions (O'connell et al., 2018). However, plasma and serum biomarkers are variable in athletes who have sustained mTBIs and may not truly reflect simultaneous alterations occurring in the brain. Moreover, recent data suggest that the glymphatic clearance system [i.e., the macroscopic waste clearance system utilizing perivascular channels to eliminate toxins and soluble proteins from the brain's interstitial space while distributing glucose, lipids and amino acids to support brain's functioning (Jessen et al., 2015)] is impaired in the acute phase of mTBI, showing a $60 \%$ decreased clearance efficiency for up to 1 month post-mTBI (Iliff et al., 2014). Although supporting evidence from human cohorts is currently lacking, it appears that glymphatic dysfunction following mTBI may result in low blood biomarker levels because of their compromised clearance (Plog and Nedergaard, 2015).

In addition, current biomarkers are not necessarily adequate to examine the long-term risk associated with mTBIs. While other potential biomarkers exist such as Positron Emission Tomography (PET), Magnetic Resonance Imaging (MRI) modalities, and computerized electroencephalogram (EEG), this review focuses solely on fluid biomarkers including exosomes. Existing fluid biomarkers have often shown inconsistent results across published studies. A number of fluid biomarkers have been examined for their ability to assess the severity of mTBI (Zetterberg et al., 2013; Papa, 2014; Papa et al., 2015; Kulbe and Geddes, 2016) but a panel consisting of multiple biomarkers may have greater sensitivity and specificity and therefore increase diagnostic accuracy (Yokobori et al., 2013; McCrea et al., 2020). Several studies suggest that exosome biomarkers may provide a consistent and sensitive assessment of ongoing brain-health issues (Fiandaca et al., 2015; Kapogiannis et al., 2019; Goetzl et al., 2019a). Therefore, we propose the use of exosomal biomarkers for assessment of PCS in athletes. This approach will also be beneficial for the much larger populations of veterans and civilians who sustain mTBIs outside of sports.

To date, there are essentially three classes of fluid biomarkers that can be used to identify mTBI: Biomarkers associated with (1) neuronal/axonal damage, (2) activation of glial cells, and (3) release of inflammatory cytokines that occur due to neuronal injury. Neuronal biomarkers that have been examined include neuron-specific enolase (NSE), calpain-derived $N$-terminal fragment of $\alpha$ II spectrin (SNTF), Tau, neurofilamentlight (NF-light), ubiquitin C-terminal hydrolase-L1 (UCH-L1), and brain-derived neurotrophic factor (BDNF). Glial activation markers include S100 calcium binding protein B (S100B) and glial fibrillary acidic protein (GFAP). Lastly, inflammatory cytokines have been measured both in CSF and blood and include the proinflammatory cytokines interleukins- $1,-12$, and -18 (IL-1, -12, 
and -18), tumor necrosis factor alpha (TNF- $\alpha$ ), and interferon gamma (IFN- $\gamma)$. Over the last few years, much attention has been brought to the development of blood biomarkers that can accurately diagnose mTBI acutely or predict the long-term changes in the brain associated with one or repeated mTBIs (Zetterberg et al., 2013; Kulbe and Geddes, 2016), and early studies have identified some promising candidates.

\section{Neuronal Biomarkers}

The first neuron-specific biomarker to be explored for mTBI was NSE (Stalnacke et al., 2004). NSE is involved in axonal transport and elevations of NSE following an injury initiates an inflammatory cascade (Kawata et al., 2016; Haque et al., 2018). Graham and colleagues showed that amateur boxers experiencing direct punches to the head had increased serum NSE levels compared to those who took punches to the body (Graham et al., 2011). Interestingly, following a 2-month resting period, serum levels of NSE remained significantly elevated in boxers compared to healthy non-boxer controls, suggesting that repetitive head trauma leads to prolonged neuronal damage (Zetterberg et al., 2009). However, a recent meta-analysis of 10 independent studies showed that NSE levels were not a strong independent predictor of PCS following mTBI (Mercier et al., 2018). Further, NSE is sensitive to hemolysis and is also present in erythrocytes and endocrine cells, and could be a potential confounder (Ramont et al., 2005).

SNTF (calpain-cleaved $\alpha$-spectrin $\mathrm{N}$-terminal fragment) has been identified as a protein that also accumulates preferentially in damaged axons, as a result of neuronal stretch injury (Johnson et al., 2016). In a study of concussed ice hockey players, Siman and colleagues reported that serum SNTF levels were increased $1 \mathrm{~h}$ following an mTBI and remained significantly elevated from $12 \mathrm{~h}$ to 6 days, before declining to pre-season baseline levels (Siman et al., 2015). SNTF is not a commonly used biomarker but might prove valuable once it has been more thoroughly investigated. In a recent meta-analysis, Gan et al. (2019) found that SNTF was a top candidate to predict delayed recovery after mTBI. Neurofilament-light (NF-light) is another biomarker for axonal damage that can be measured in serum. NF-light levels are increased in ice hockey players with PCS (Shahim et al., 2017) and in active professional fighters as well as in retired fighters with neurological issues (Bernick et al., 2018). However, no difference in serum NF-light levels were found between baseline and 6-day and 14-day post mTBI in a cohort of adolescent athletes with uncomplicated mTBI, suggesting that the severity of the neuronal injury might have been too low to detect changes in NF-light levels (Wallace et al., 2018).

Ubiquitin carboxy-terminal hydrolase L1 (UCH-L1) is an enzyme involved both in the processing of ubiquitin precursors and of ubiquitinated proteins and is highly expressed in the neuronal cytoplasm (Setsuie and Wada, 2007). Serum UCHL1 has a high accuracy albeit with a high risk of bias in predicting cat scan (CT) findings in mTBI and appears as a promising blood-based diagnostic biomarker for TBI, but further studies are needed (Shahjouei et al., 2018). UCH-L1 interacts with both $\mathrm{p}$-Tau and $\mathrm{A} \beta$, making the ubiquitin pathway a promising target for development of novel treatment avenues for neurodegenerative disease (Papa et al., 2015; Joseph et al., 2018; McCrea et al., 2020). McCrea and colleagues (McCrea et al., 2020) recently showed that serum UCH-L1 levels were acutely elevated in concussed athletes compared to baseline preseason levels but that levels had returned to baseline $48 \mathrm{~h}$ after injury or 7 days after RTP. It was recently reported significant elevations in UCH-L1 levels in neuron-derived exosomes (NDEs) obtained from athletes with acute, but not chronic, mTBIs suggesting that this marker may be an excellent predictor of short-term effects (Goetzl et al., 2019a).

Gill et al. (2017) showed that, in collegiate athletes, increased plasma total Tau concentration within $6 \mathrm{~h}$ of mTBI correlated to having an extended RTP duration. In a recent study of collegiate football players, the investigators found significant elevations in plasma Tau levels post-practice, but no correlation between the number of sub-concussions or mTBIs and Tau (Kawata et al., 2016). Alosco et al. (2018) found that greater exposure to repetitive head impacts in a cohort of 96 former NFL players correlated with higher plasma total Tau concentrations later in life. Pathological phosphorylation of Tau has been associated with long-term risk for neurodegenerative disease, such as CTE after repeated mTBIs (Baugh et al., 2015; Daneshvar et al., 2015; Asken et al., 2016; Di Battista et al., 2016; Broglio et al., 2017; Alosco et al., 2018; Barnes et al., 2018; Goetzl et al., 2019b). p-Tau is considered an important biomarker for determining long-term effects of mTBIs in terms of risk for developing dementias because neuropathological findings confirm that $\mathrm{p}$-Tau accumulates in the brain within NFTs in both AD and in CTE (Tracy and Gan, 2018). Our recent findings indicate that levels of p-Tau (T181 and S396) exhibit long-term changes when measured in NDE preps from blood (Goetzl et al., 2019a), strongly suggesting that this novel method for assessing biomarkers in exosomes of neuronal origin may provide an important insight into brain-specific events after injury. Although these are highly experimental procedures to date, they hold promise for development of successful targeting of Tau pathology in the near future.

Brain-derived neurotrophic factor (BDNF) is a neurotrophic factor highly involved in brain health, with serum levels increasing after physical exercise or cognitive training (Hakansson et al., 2017; Ledreux et al., 2019). It has been suggested that BDNF-related treatments could offer neuroprotection and help facilitate neuroplastic changes to reverse deficits associated with TBI (Kaplan et al., 2010). Korley et al. (2016) have found decreased day-of-injury BDNF levels after TBI compared to non-TBI controls.

\section{Glial Activation Biomarkers}

Astroglial proteins such as $\mathbf{S 1 0 0}$ calcium-binding protein B (S100B) and glial fibrillary acidic protein (GFAP) are proteins located in the cytoplasm of astrocytes and have received a lot of attention as markers of glial activation, particularly following mTBIs (Kawata et al., 2016; Sajja et al., 2016; Bogoslovsky et al., 2017). The levels of S100B can help differentiate patients with mTBI from those with severe TBI and was shown to improve predictions of long-term post-concussion outcomes (Dey et al., 2017). However, there is evidence that S100B is not only produced in the brain but also in peripheral cells such as adipocytes 
and chondrocytes (Diaz-Romero et al., 2014), thus making its measurement in peripheral blood less specific to sport-related mTBI and providing a rationale for focusing on other glial biomarkers which could measure more directly effects of mTBIs associated with brain damage.

Neselius et al. (2012) showed that CSF levels of GFAP were increased within 6 days after an mTBI in more than $80 \%$ of a cohort of 30 Olympic boxers and returned to levels comparable to the control cohort after a rest period of 14 days. Interestingly, there are conflicting reports regarding the efficiency of GFAP as a biomarker in terms of predictive and diagnostic value for post-mTBI assessments. While several research groups found that GFAP was the most promising biomarker in terms of serum elevations after mTBI (Kou et al., 2013), others did not see significant increases in GFAP, even in athletes with mTBIs (Meier et al., 2017). Others have shown, using singlemolecule array (Simoa) technology, that individuals with TBI exhibit elevated levels of GFAP immediately after arrival in the hospital (Bogoslovsky et al., 2017). In a recent study, McCrea and colleagues showed that athletes with mTBI had significantly elevated levels of GFAP compared to pre-season baseline levels, and that a more severe mTBI with loss of consciousness resulted in further elevated GFAP levels, thus suggesting that this biomarker can be of potential clinical utility (McCrea et al., 2020). Both glial markers GFAP and S100B are still among the most investigated biomarkers for $\mathrm{mTBI}$, especially for short-term effects and for diagnostics in the emergency room following an injury. However, one should keep in mind the potential contribution of the glymphatic clearance system since preclinical data obtained in a murine TBI model demonstrated that inhibition of the glymphatic clearance (Jessen et al., 2015) suppressed TBI-induced increased serum levels of S100B and GFAP (Plog et al., 2015).

\section{Inflammatory Cytokines}

Release of inflammatory cytokines reflects the activation of glial cells in the brain, as well as increased activation of the peripheral immune system which can also indirectly affect neuroinflammation (Patterson and Holahan, 2012). mTBI is associated with neuroinflammation, expressed as elevated proand anti-inflammatory cytokines in both CSF and in blood (Hobbs et al., 2016; Singh et al., 2016). Neuroinflammation, which is a natural response to widespread injury in the brain, can be detrimental for neuronal survival and increases the activation of microglia and astrocytes in the vicinity of the injury. The severity of this secondary wave of injury, caused by inflammation and activation of glial cells, is highly influential on long-term outcomes (e.g., Jassam et al., 2017). In the subacute period after sport-related mTBI, Di Battista and colleagues found increased levels of MCP- 4 and MIP- $1 \beta$ that could discriminate between athletes with mTBI compared to healthy controls in the acute phase post-injury, however, no significant differences were observed at the time of medical clearance (Di Battista et al., 2016). These authors also found that subacute levels of MCP1 and MCP-4 were positively correlated with days to recovery in athletes with mTBI. In another study, symptom duration and IL-6 levels $6 \mathrm{~h}$ post-mTBI were significantly associated in high school and collegiate football players (Nitta et al., 2019), thus highlighting the clinical significance of inflammatory markers, at least for the acute phase after mTBI. While a limited number of studies have investigated the changes in inflammatory markers after mTBI, examination of inflammatory markers in neuron-, and astrocyte-derived exosomes (ADEs) could further our understanding of the neurophysiological consequences of sport-related mTBI since neuroinflammation appears to be a key player in neurodegenerative processes.

\section{Exosomal Biomarkers}

As described above, blood levels of NF-light, GFAP, UCH-L1, $\mathrm{Tau} / \mathrm{p}$-Tau, and/or inflammatory biomarkers are promising for identifying the acute phase of mTBI but it has been shown that blood biomarkers have limitations for quantifying the chronic effects of repeated mTBIs (Agoston et al., 2017). On the contrary, CSF biomarkers can better quantify post-acute effects and inform the time line for RTP (Papa et al., 2015) but both animal and human studies of mTBI demonstrate the importance of serial sampling after a concussive event, which makes it difficult to rely on CSF biomarkers in clinical situations. Therefore, the identification of exosomal biomarkers involved in post-acute PCS could contribute significantly to the identification, diagnosis, and prognosis of the underlying individual pathobiological changes of mTBI and the long-term effects of repeated mTBIs.

Exosomes are nanosized extracellular vesicles that are produced by every cell type in the body and are released for degradation of cargo and/or signaling/transportation from cell to cell (Hamlett et al., 2019). By measuring total Tau levels in total exosomes from plasma, Stern and collaborators were able to discern between CTE and control patients more than $80 \%$ of the time. Interestingly, higher levels of exosomal tau were associated with worse performance on memory tests, but not with mood or behavior deficits (Stern et al., 2016). This correlation was not present when measuring total Tau in plasma, although this measure was positively associated with cumulative head impact index (Alosco et al., 2018). Guedes et al. (2020) measured plasma and exosomal levels of NF-light in veterans with history of mTBIs and found that those experiencing chronic PCS and posttraumatic stress disorder (PTSD) exhibited the highest NF-light levels both in plasma and exosomes.

The analysis of CNS-derived exosomes by ultra-high sensitivity detection methods is a novel method that has the potential to provide ongoing information regarding brain health both in athletes and service members exposed to one or several mTBIs. To our knowledge, Gill et al. (2018) were the first to demonstrate the effects of mTBIs on exosomal biomarkers. They utilized ultrasensitive single molecule array (Simoa) technology to measure Tau, $A \beta 42$, and IL-10 levels in NDE from 42 military personnel with mTBI compared to 22 military with no mTBI. They found that NDE Tau levels were elevated following mTBIs compared to those with no mTBI (Gill et al., 2018). Further, they observed that within the mTBI group, post-concussive symptoms were most related to NDE Tau elevations, whereas NDE IL-10 levels were related to PTSD symptoms. This study strongly suggested that NDE biomarkers may be more sensitive 
and may also better reflect post-acute changes in the brain postconcussion than blood biomarkers. However, more experiments are needed to determine whether total Tau or p-Tau levels in CNS-derived exosomes can predict long-term conversion to CTE or other tauopathies in individuals with repeated mTBIs.

In a recent study by our group (Goetzl et al., 2019a), differential exosomal increases of certain proteins acutely following an mTBI (annexin VII, UCH-L1, claudin-5, aquaporin 4 , and synaptogyrin-3), and chronically in student athletes with chronic mTBIs [ $>12$ months prior; A $\beta 42$, p-tau T181, p-tau S396, IL-6, and cellular prion protein (PRPc)] suggested a shift in pathological processes as a result of time after injury. Although these findings are promising, more studies are needed that examine exosomal cargo changes long-term after repeated mTBIs, as would be the case for student athletes playing highimpact sports.

Other content of exosomes, particularly their miRNA cargo, can impact the post-transcriptional regulation of many genes. miRNAs are small non-coding, single-stranded RNA molecules comprising of around 22 nucleotides (Zhang et al., 2015). miRNA carried by exosomes are involved in various key processes such as nerve and vascular regeneration and can either promote regeneration or aggravate degeneration (Kalani et al., 2014). Although miRNAs function in the brain is still being investigated, altered miRNA levels in blood and saliva following TBI have been reported, and several candidates miRNA have been identified as potential biomarkers for TBI (Atif and Hicks, 2019). Recently, using a combination of microchip diagnostic and machine learning, Ko et al. (2018) have developed a platform to isolate brain-derived circulating exosomes and measure multiple miRNAs packaged within these exosomes to accurately identify TBI patients from healthy controls. New treatment paradigms involving miRNA are also being explored and would be especially promising if it can be proven that a single miRNA has the ability to influence several target genes, making it possible for the researchers to potentially modify a whole disease phenotype by modulating a single miRNA molecule.

\section{Decreased Exosome Release After TBI}

Concentrations of NDEs, assessed by counts and levels of exosome marker CD81, were significantly depressed by a mean of $45 \%$ after acute mTBI in collegiate athletes, but not in student athletes with chronic mTBI, compared with controls (Goetzl et al., 2019a). A recent manuscript also showed that the levels of ADEs were lower immediately after a severe TBI, but levels of ADEs returned to normal by 12 months post-TBI (Goetzl et al., 2020). On the other hand, Winston et al. (2019b) found no differences in CD81 levels between veterans with or without history of mTBI, highlighting the need for more research to examine the potential effects of timing of blood sampling after mTBI and source of exosomes as factors that could affect the exosome release. Others have shown that microvesicles released in the CSF from people with TBI had a smaller diameter than in controls (Manek et al., 2018).

Mechanisms involved in these observations may be related to increased autophagy occurring after mTBIs or TBIs, as demonstrated by Smith et al. (2011) and illustrated by the schematic drawing depicted in Figure 2. A primary reason may be the increase in autophagy reported acutely after brain and spinal injury (Smith et al., 2011), perhaps leading to reduced exosomal production by default (Figure 2). Smith and collaborators propose that reduced exosomal release could be a physiological response, recycling injured organelles and other products of deterioration instead of releasing them into multivesicular bodies and exosomes. Relative levels of the autophagy marker LC3-II were increased by 100-200\% in injured rat brains compared with sham rats (Liu et al., 2008), supporting this notion. LC3 immunoreactivity is also increased severalfold in rats after controlled cortical impact compared with sham injury, peaking at 8 days (Zhang et al., 2008). Other
A Normal function

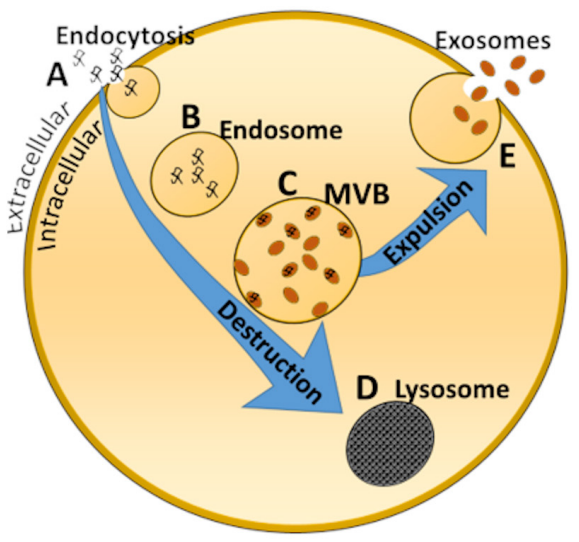

B Following TBI

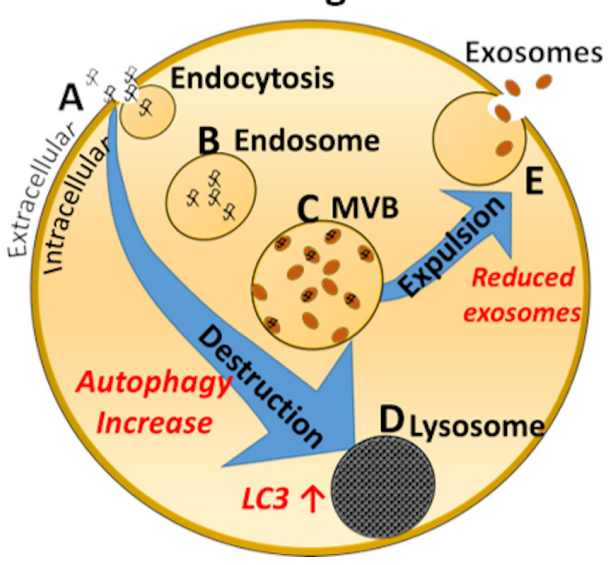

FIGURE 2 | Schematic representation of exosome biology in a normal cell (A) and after mTBI (B). Normally, endocytosis (A) leads to inclusions of early endosomes (B), which in turn leads to development of multivesicular bodies (MVB, C), which can either be demolished by the lysosomal pathway (D), or expelled from the cell as exosomes (E). Studies suggest that after a TBI the lysosomal/autophagosomal pathway is increased, to the detriment of exosomal secretion. Autophagosomal markers, such as LC3 are elevated. 
studies (Chen et al., 2018) suggest that inducing autophagy (for example by using Omega-3 supplements) might be beneficial for recovery after mTBIs, suggesting that elevated autophagy is a repair mechanism in the brain. The alteration in the exosomal/autophagy pathway after TBI or mTBI is diametrically different to that occurring for example in people with Down syndrome, where the levels of autophagy are reduced and levels of the exosomal marker CD63 are increased manifested by increased exosomal release (Hamlett et al., 2019). Of course, there are many pathological events that occur, both short- and long-term, after mTBIs that contribute to secondary damage, poor outcome and future increased risk for neurodegenerative conditions. For example, future research should focus on addressing how the blood-brain barrier integrity and glymphatic system dysfunction that have been described after TBI affect exosome production and release (Sullan et al., 2018; O'keeffe et al., 2019). Because regulation of autophagy can exhibit antioxidative stress, anti-apoptosis and anti-inflammatory effects following repeated mTBIs, this represents a promising target for further therapeutic development (Zhang and Wang, 2018). In addition, many neuroprotective drugs may attenuate TBIinduced secondary brain injury via activation of autophagy (Ding et al., 2015; Gao et al., 2017; Zhang et al., 2017).

\section{POTENTIAL USE OF EXOSOMES FOR TREATMENT OF TBI}

Novel data from several research groups strongly suggest that exosomes may serve not only as a biomarker source, but also as a delivery tool of novel treatment therapies for TBIs and mTBIs (Figure 3A). Xiong et al. (2017b) posed that exosomes are more stable and can cross the blood-brain barrier, compared with their parent cells. When administering, for example, mesenchymal stem cells (MSCs), there are major safety risks associated with the risk of occlusion in microvasculature or unregulated growth of transplanted cells in brain parenchyma (Xiong et al., 2017b). Therefore, the development of cell-free exosome-based therapy may provide a novel approach to enhancing neuroplasticity and amplifying neurological recovery following one or several mTBIs (see Figure 3A). Beneficial effects of exosomes derived from stem cells in rodent models for TBI have been demonstrated (Yang et al., 2017; Zhang et al., 2019). A huge advantage with these stem cell-derived exosomes is that they could be delivered systemically, reducing the need for more invasive treatment avenues. Li et al. (2017) suggested that the beneficial effects afforded by stem cellderived exosomes on TBI recovery in a rat model were at least partially due to a shift in microglial phenotype from the M1 to the M2 phenotype, strongly indicating that inflammation plays a major role for long-term outcomes after TBIs. Finally, Kaijzel et al. (2017) suggest that nanoparticles, including exosomes and other cell-free delivery systems, may provide a more targeted approach for successful intervention and prevention therapies, to prevent long-term brain damage following one or several mTBIs. However, this promising field is yet in its infancy, although the cancer field has reached much further in the search for exosomebased therapies (Weston et al., 2019). Most likely, the TBI field

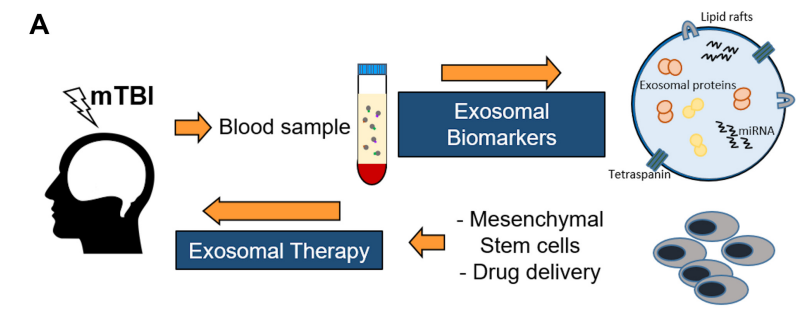

B

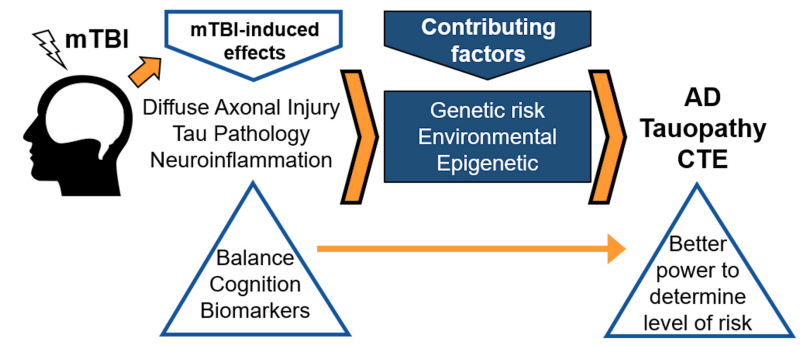

FIGURE 3 | Schematic showing the use of exosomes both as biomarkers and therapeutic agents (A), and a proposed protocol for genetic, epigenetic, and environmental factors involved in post-concussion outcomes in humans (B)

(A) Purifying either ADEs or NDEs from blood samples can lead to identification of brain-related factors, both proteins and miRNAs, that are involved in long-term or short-term post-concussion brain alterations. In addition, recent work from the cancer field suggests that exosomes obtained from enriched stem cells, such as mesenchymal stem cells, can be delivered in blood to reach and benefit brain areas affected by TBI. (B) Mild traumatic brain injury (mTBI) induces several adverse pathological effects in the brain. These effects, along with genetic, environmental, and epigenetic factors, contribute to an enhanced risk of neurodegeneration later in life.

Neurodegeneration, as a result of the damage produced by mTBI, may later present itself in the form of Alzheimer's disease (AD), tauopathy, chronic traumatic encephalopathy (CTE), and other maladies. Genetic, epigenetic, as well as environmental factors may affect the outcome after mTBls. However, it may be possible to identify those individuals at increased risk of

neurodegeneration due to $\mathrm{mTBI}$ by incorporating a more rigorous assessment using enhanced balance and cognitive testing along with biomarker studies following mTBI.

can benefit from the findings in the cancer field and spur the development of promising new therapies.

\section{SUMMARY AND CONCLUSION}

A single or repeated mTBI can cause short-term symptoms (collectively called PCS) and may also increase the risk for long-term neurodegenerative conditions including CTE, AD, or other neurodegenerative or neuropsychiatric conditions. Ongoing research suggests that current RTP protocols should evolve to include quantitative assessments of reported symptoms, cognition, vestibulo-ocular function, dynamic balance, and exosomal biomarkers. The expansion of that battery will confer a direct benefit to the athletic community and increase the safety of RTP protocols. It will also contribute to concussion management education and awareness as well as earlier detection of symptoms that may be related to long-term neurodegenerative disease. 
Several studies have suggested that a battery including selfreported symptoms as well as a neurocognitive and balance tasks have a sensitivity to concussion exceeding 90\%. However, most studies have not considered long-term PCS changes in the brain that may impact brain health. Therefore, it appears important to obtain and bank baseline data on all athletes. That includes individualized testing for balance, cognitive performance, and biomarkers. Our experience from a cohort consisting of more than 300 NCAA Division I athletes in high-impact sports clearly shows a need for expanded testing paradigms including instrumented balance measurements and better cognitive batteries that capture long-term changes in executive function, impulse control, and decision making, for example supplementing the ImPACT battery with portions of the ANAM test battery.

Although more research is needed to determine the most sensitive and specific biomarkers for long-term brain health, it seems appropriate to include a panel of biomarkers in mTBI protocols in cases where PCS appears (Gan et al., 2019; McCrea et al., 2020). Further, our data presented herein strongly suggest that exosomal biomarkers may represent a more sensitive and reliable biomarker method, at least for long-term effects. Our recent findings indicate that Tau, p-Tau S396, p-Tau T181, and $\mathrm{A} \beta 42$ exhibit long-term changes when measured in NDE preps from blood (Goetzl et al., 2019a), strongly suggesting that this novel method for assessing biomarkers in exosomes of neuronal origin may provide an important insight into brain-specific events after injury. Based on our findings and those by others, it is proposed herein that exosomes can be used both as a valuable "liquid biopsy" to determine what goes on in the brain - either glial cells or neurons - and can also be developed to a novel way to treat mTBIs, for example using exosomes derived from MSCs

\section{REFERENCES}

Agoston, D., Shutes-David, A., and Peskind, E. (2017). Biofluid biomarkers of traumatic brain injury. Brain Inj. 31, 1195-1203. doi: 10.1080/02699052.2017. 1357836

Akin, F. W., Murnane, O. D., Hall, C. D., and Riska, K. M. (2017). Vestibular consequences of mild traumatic brain injury and blast exposure: a review. Brain Inj. 31, 1188-1194. doi: 10.1080/02699052.2017.1288928

Albayram, O., Kondo, A., Mannix, R., Smith, C., Tsai, C. Y., Li, C., et al. (2017). Cis P-tau is induced in clinical and preclinical brain injury and contributes to post-injury sequelae. Nat. Commun. 8:1000. doi: 10.1038/s41467-017-01068-4

Alla, S., Sullivan, S. J., McCrory, P., and Hale, L. (2011). Spreading the word on sports concussion: citation analysis of summary and agreement, position and consensus statements on sports concussion. Br. J. Sports Med. 45, 132-135. doi: $10.1136 /$ bjsm.2010.074088

Allum, J. H., and Honegger, F. (2016). Recovery times of stance and gait balance control after an acute unilateral peripheral vestibular deficit. J. Vestib. Res. 25, 219-231. doi: 10.3233/VES-150561

Alosco, M. L., Tripodis, Y., Fritts, N. G., Heslegrave, A., Baugh, C. M., Conneely, S., et al. (2018). Cerebrospinal fluid tau, Abeta, and sTREM2 in former national football league players: modeling the relationship between repetitive head impacts, microglial activation, and neurodegeneration. Alzheimers Dement 14, 1159-1170. doi: 10.1016/j.jalz.2018.05.004

Armstrong, R. A., McKee, A. C., Alvarez, V. E., and Cairns, N. J. (2017). Clustering of tau-immunoreactive pathology in chronic traumatic encephalopathy. J. Neural Transm. (Vienna) 124, 185-192. doi: 10.1007/s00702-016-1635-1
(Mondello et al., 2018; Figure 3). Animal and in vitro studies can reveal biological mechanisms involved in disease progress longterm, as discussed above. Based on previous and current findings in the field, a model is emerging in which diffuse axonal injury, Tau pathology, and neuroinflammation incrementally contribute to long-term changes in the brain that eventually lead to increased risk for tauopathies such as $\mathrm{AD}$ or CTE with aging.

\section{AUTHOR CONTRIBUTIONS}

A-CG, AL, DL, KG, and BD contributed to the conception of the review, coordinated writing efforts, and edited final article version. A-CG, AL, and KG wrote the Introduction. MP and BD wrote the balance assessment part. KG prepared the cognitive assessment part. AL and A-CG wrote the biomarker and exosome portions. DL, LK, and HF worked on the animal studies section. All authors collaborated with or were part of the DU Concussion research group and contributed to critical revisions of the manuscript, read, and approved the submitted version.

\section{FUNDING}

This work was funded by an internal KIHA pilot grant to $\mathrm{DL}, \mathrm{BD}$, and $\mathrm{KG}$.

\section{ACKNOWLEDGMENTS}

The authors would like to acknowledge the technical expertise of Hammam Belgasem for his work on the DU Concussion Study.

Asken, B. M., Sullan, M. J., Snyder, A. R., Houck, Z. M., Bryant, V. E., Hizel, L. P., et al. (2016). Factors influencing clinical correlates of chronic traumatic encephalopathy (CTE): a review. Neuropsychol. Rev. 26, 340-363. doi: 10.1007/ s11065-016-9327-z

Atif, H., and Hicks, S. (2019). A review of microRNA biomarkers in traumatic brain injury. J. Exp. Neurosci. 13, 1-12.

Barnes, D. E., Byers, A. L., Gardner, R. C., Seal, K. H., Boscardin, W. J., and Yaffe, K. (2018). Association of mild traumatic brain injury with and without loss of consciousness with dementia in US Military veterans. JAMA Neurol. 75, 1055-1061. doi: 10.1001/jamaneurol.2018.0815

Baugh, C. M., Kroshus, E., Daneshvar, D. H., Filali, N. A., Hiscox, M. J., and Glantz, L. H. (2015). Concussion management in United States college sports: compliance with National Collegiate Athletic Association concussion policy and areas for improvement. Am. J. Sports Med. 43, 47-56. doi: 10.1177/ 0363546514553090

Bennett, R. E., Mac Donald, C. L., and Brody, D. L. (2012). Diffusion tensor imaging detects axonal injury in a mouse model of repetitive closed-skull traumatic brain injury. Neurosci. Lett. 513, 160-165. doi: 10.1016/j.neulet.2012.02.024

Bernick, C., Zetterberg, H., Shan, G., Banks, S., and Blennow, K. (2018). Longitudinal performance of plasma neurofilament light and tau in professional fighters: the professional fighters brain health study. J. Neurotrauma 35, 23512356. doi: 10.1089/neu.2017.5553

Berz, K., Divine, J., Foss, K. B., Heyl, R., Ford, K. R., and Myer, G. D. (2013). Sexspecific differences in the severity of symptoms and recovery rate following sports-related concussion in young athletes. Phys. Sportsmed. 41, 58-63. doi: 10.3810/psm.2013.05.2015 
Bogoslovsky, T., Wilson, D., Chen, Y., Hanlon, D., Gill, J., Jeromin, A., et al. (2017). Increases of plasma levels of glial fibrillary acidic protein, tau, and amyloid beta up to 90 days after traumatic brain injury. J. Neurotrauma 34, 66-73. doi: 10.1089/neu.2015.4333

Bramley, H., Hong, J., Zacko, C., Royer, C., and Silvis, M. (2016). Mild traumatic brain injury and post-concussion syndrome: treatment and related sequela for persistent symptomatic disease. Sports Med. Arthrosc Rev. 24, 123-129. doi: 10.1097/JSA.0000000000000111

Broglio, S. P., McCrea, M., McAllister, T., Harezlak, J., Katz, B., Hack, D., et al. (2017). A national study on the effects of concussion in collegiate athletes and US military service academy members: the NCAA-DoD concussion assessment, research and education (CARE) consortium structure and methods. Sports Med. 47, 1437-1451. doi: 10.1007/s40279-017-0707-1

Buckley, T. A., Oldham, J. R., Watson, D. J., Murray, N. G., Munkasy, B. A., and Evans, K. M. (2019). Repetitive head impacts in football do not impair dynamic postural control. Med. Sci. Sports Exerc. 51, 132-140. doi: 10.1249/ MSS.0000000000001761

Cantu, R. C. (1997). Athletic head injuries. Clin. Sports Med. 16, 531-542. doi: 10.1016/s0278-5919(05)70038-7

Carman, A. J., Ferguson, R., Cantu, R., Comstock, R. D., Dacks, P. A., Dekosky, S. T., et al. (2015). Expert consensus document: Mind the gaps-advancing research into short-term and long-term neuropsychological outcomes of youth sports-related concussions. Nat. Rev. Neurol. 11, 230-244. doi: 10.1038/ nrneurol.2015.30

Carson, A. (2017). Concussion, dementia and CTE: are we getting it very wrong? J. Neurol. Neurosurg. Psychiatry 88, 462-464. doi: 10.1136/jnnp-2016-315510

Cecil, K. M., Hills, E. C., Sandel, M. E., Smith, D. H., McIntosh, T. K., Mannon, L. J., et al. (1998). Proton magnetic resonance spectroscopy for detection of axonal injury in the splenium of the corpus callosum of brain-injured patients. J. Neurosurg. 88, 795-801. doi: 10.3171/jns.1998.88.5.0795

Chen, C. J., and Chou, L. S. (2010). Center of mass position relative to the ankle during walking: a clinically feasible detection method for gait imbalance. Gait Posture 31, 391-393. doi: 10.1016/j.gaitpost.2009.11.010

Chen, H., Desai, A., and Kim, H. Y. (2017). Repetitive closed-head impact model of engineered rotational acceleration induces long-term cognitive impairments with persistent astrogliosis and microgliosis in mice. J. Neurotrauma 34, 22912302. doi: 10.1089/neu.2016.4870

Chen, X., Pan, Z., Fang, Z., Lin, W., Wu, S., Yang, F., et al. (2018). Omega-3 polyunsaturated fatty acid attenuates traumatic brain injury-induced neuronal apoptosis by inducing autophagy through the upregulation of SIRT1-mediated deacetylation of Beclin-1. J. Neuroinflamm. 15:310. doi: 10.1186/s12974-0181345-8

Choe, M. C., and Giza, C. C. (2015). Diagnosis and management of acute concussion. Semin. Neurol. 35, 29-41. doi: 10.1055/s-0035-1544243

Collins, C. L., Fletcher, E. N., Fields, S. K., Kluchurosky, L., Rohrkemper, M. K., Comstock, R. D., et al. (2014). Neck strength: a protective factor reducing risk for concussion in high school sports. J. Prim. Prev. 35, 309-319. doi: 10.1007/ s10935-014-0355-2

Collins-Praino, L. E., and Corrigan, F. (2017). Does neuroinflammation drive the relationship between tau hyperphosphorylation and dementia development following traumatic brain injury? Brain Behav. Immun. 60, 369-382. doi: 10. 1016/j.bbi.2016.09.027

Cottle, J. E., Hall, E. E., Patel, K., Barnes, K. P., and Ketcham, C. J. (2017). Concussion baseline testing: preexisting factors, symptoms, and neurocognitive performance. J. Athl. Train 52, 77-81. doi: 10.4085/1062-6050-51.12.21

Covassin, T., Elbin, R., and Stiller-Ostrowski, J. L. (2009). Current sport-related concussion teaching and clinical practices of sports medicine professionals. J. Athl. Train 44, 400-404. doi: 10.4085/1062-6050-44.4.400

Covassin, T., Schatz, P., and Swanik, C. B. (2007). Sex differences in neuropsychological function and post-concussion symptoms of concussed collegiate athletes. Neurosurgery 61, 345-350. doi: 10.1227/01.NEU. 0000279972.95060.CB discussion 350-341.

Cruz-Haces, M., Tang, J., Acosta, G., Fernandez, J., and Shi, R. (2017). Pathological correlations between traumatic brain injury and chronic neurodegenerative diseases. Transl. Neurodegener 6:20.

Daneshvar, D., Riley, D., Nowinski, C., McKee, A., Stern, R., and Cantu, R. (2011). Long-term consequences: effects on normal development profile after concussion. Phys. Med. Rehabil. Clin. N. Am. 22, 683-700. doi: 10.1016/j.pmr. 2011.08.009

Daneshvar, D. H., Goldstein, L. E., Kiernan, P. T., Stein, T. D., and McKee, A. C. (2015). Post-traumatic neurodegeneration and chronic traumatic encephalopathy. Mol. Cell. Neurosci. 66, 81-90. doi: 10.1016/j.mcn.2015.03.007

Deleo, A. M., and Ikezu, T. (2018). Extracellular vesicle biology in alzheimer's disease and related tauopathy. J. Neuroimmune Pharmacol. 13, 292-308. doi: 10.1007/s11481-017-9768-z

Dey, S., Gangadharan, J., Deepika, A., Kumar, J., Christopher, R., Ramesh, S., et al. (2017). Correlation of ubiquitin $C$ terminal hydrolase and $S 100 \beta$ with cognitive deficits in young adults with mild traumatic brain injury. Neurol. India 65, 761-766.

Di Battista, A. P., Rhind, S. G., Richards, D., Churchill, N., Baker, A. J., and Hutchison, M. G. (2016). Altered blood biomarker profiles in athletes with a history of repetitive head impacts. PLoS ONE 11:e0159929. doi: 10.1371/journal. pone.0159929

Diaz-Romero, J., Quintin, A., Schoenholzer, E., Pauli, C., Despont, A., Zumstein, M., et al. (2014). S100A1 and S100B expression patterns identify differentiation status of human articular chondrocytes. J. Cell. Physiol. 229, 1106-1117. doi: $10.1002 /$ jcp. 24547

Ding, K., Xu, J., Wang, H., Zhang, L., Wu, Y., and Li, T. (2015). Melatonin protects the brain from apoptosis by enhancement of autophagy after traumatic brain injury in mice. Neurochem. Int. 91, 46-54. doi: 10.1016/j.neuint.2015. 10.008

Echemendia, R. J., Meeuwisse, W., McCrory, P., Davis, G. A., Putukian, M., Leddy, J., et al. (2017). The sport concussion assessment tool 5th Edition (SCAT5): background and rationale. Br. J. Sports Med. 51, 848-850. doi: 10.1136/bjsports2017-097506

Edwards, G., Moreno-Gonzalez, I., and Soto, C. (2017). Amyloid-beta and tau pathology following repetitive mild traumatic brain injury. Biochem. Biophys. Res. Commun. 483, 1137-1142. doi: 10.1016/j.bbrc.2016.07.123

Falcon, B., Zivanov, J., Zhang, W., Murzin, A. G., Garringer, H. J., Vidal, R., et al. (2019). Novel tau filament fold in chronic traumatic encephalopathy encloses hydrophobic molecules. Nature 568, 420-423. doi: 10.1038/s41586-019-1026-5

Fiandaca, M. S., Kapogiannis, D., Mapstone, M., Boxer, A., Eitan, E., Schwartz, J. B., et al. (2015). Identification of preclinical Alzheimer's disease by a profile of pathogenic proteins in neurally derived blood exosomes: a case-control study. Alzheimers Dement 11, 600-607.e601. doi: 10.1016/j.jalz.2014.06.008

Finnoff, J. T., Peterson, V. J., Hollman, J. H., and Smith, J. (2009). Intrarater and interrater reliability of the Balance Error Scoring System (BESS). PMR 1, 50-54. doi: 10.1016/j.pmrj.2008.06.002

Fitzpatrick, A. W. P., Falcon, B., He, S., Murzin, A. G., Murshudov, G., Garringer, H. J., et al. (2017). Cryo-EM structures of tau filaments from Alzheimer's disease. Nature 547, 185-190. doi: 10.1038/nature23002

Furman, J. L., Vaquer-Alicea, J., White, C. L., Cairns, N. J., Nelson, P. T., and Diamond, M. I. (2017). Widespread tau seeding activity at early Braak stages. Acta Neuropathol. 133, 91-100. doi: 10.1007/s00401-016-1644-z

Galetta, K. M., Barrett, J., Allen, M., Madda, F., Delicata, D., Tennant, A. T., et al. (2011). The King-Devick test as a determinant of head trauma and concussion in boxers and MMA fighters. Neurology 76, 1456-1462. doi: 10.1212/WNL. $0 \mathrm{~b} 013 \mathrm{e} 31821184 \mathrm{c} 9$

Gan, Z. S., Stein, S. C., Swanson, R., Guan, S., Garcia, L., Mehta, D., et al. (2019). Blood biomarkers for traumatic brain injury: a quantitative assessment of diagnostic and prognostic accuracy. Front. Neurol. 10:446. doi: 10.3389/fneur. 2019.00446

Gao, Y., Zhuang, Z., Gao, S., Li, X., Zhang, Z., Ye, Z., et al. (2017). Tetrahydrocurcumin reduces oxidative stress-induced apoptosis via the mitochondrial apoptotic pathway by modulating autophagy in rats after traumatic brain injury. Am. J. Transl. Res. 9, 887-899.

Gardner, R. C., Byers, A. L., Barnes, D. E., Li, Y., Boscardin, J., and Yaffe, K. (2018). Mild TBI and risk of parkinson disease: a chronic effects of neurotrauma consortium study. Neurology 90, e1771-e1779. doi: 10.1212/ WNL.0000000000005522

Gil, J. A., Defroda, S. F., Kriz, P., and Owens, B. D. (2017). Epidemiology of snow skiing- versus snowboarding-related concussions presenting to the emergency department in the united states from 2010 to 2014. Clin. J. Sport. Med. 27, 499-502. doi: 10.1097/JSM.0000000000000395 
Gill, J., Merchant-Borna, K., Jeromin, A., Livingston, W., and Bazarian, J. (2017). Acute plasma tau relates to prolonged return to play after concussion. Neurology 88, 595-602. doi: 10.1212/WNL.0000000000003587

Gill, J., Mustapic, M., Diaz-Arrastia, R., Lange, R., Gulyani, S., Diehl, T., et al. (2018). Higher exosomal tau, amyloid-beta 42 and IL-10 are associated with mild TBIs and chronic symptoms in military personnel. Brain Inj. 32, 12771284. doi: 10.1080/02699052.2018.1471738

Giza, C. C., Kutcher, J. S., Ashwal, S., Barth, J., Getchius, T. S., Gioia, G. A., et al. (2013). Summary of evidence-based guideline update: evaluation and management of concussion in sports: report of the Guideline Development Subcommittee of the American Academy of Neurology. Neurology 80, 2250 2257. doi: 10.1212/WNL.0b013e31828d57dd

Goetzl, E. J., Elahi, F. M., Mustapic, M., Kapogiannis, D., Pryhoda, M., Gilmore, A., et al. (2019a). Altered levels of plasma neuron-derived exosomes and their cargo proteins characterize acute and chronic mild traumatic brain injury. FASEB J. 33, 5082-5088. doi: 10.1096/fj.201802319R

Goetzl, E. J., Ledreux, A., Granholm, A. C., Elahi, F. M., Goetzl, L., Hiramoto, J., et al. (2019b). Neuron-derived exosome proteins may contribute to progression from repetitive mild traumatic brain injuries to chronic traumatic encephalopathy. Front Neurosci 13:452. doi: 10.3389/fnins.2019.00452

Goetzl, E. J., Yaffe, K., Peltz, C. B., Ledreux, A., Gorgens, K., Davidson, B., et al. (2020). Traumatic brain injury increases plasma astrocyte-derived exosome levels of neurotoxic complement proteins. FASEB J. 34, 3359-3366. doi: 10. 1096/fj.201902842R

Gozt, A., Licari, M., Halstrom, A., Milbourn, H., Lydiard, S., Black, A., et al. (2020). Towards the development of an integrative, evidence-based suite of indicators for the prediction of outcome following mild traumatic brain injury: results from a pilot study. Brain Sci. 10:23. doi: 10.3390/brainsci10010023

Graham, M., Myers, T., Evans, P., Davies, B., Cooper, S., Bhattacharya, K., et al. (2011). Direct hits to the head during amateur boxing is associated with a rise in serum biomarkers for brain injury. Int. J. Immunopathol. Pharmacol. 24, 119-125. doi: 10.1177/039463201102400114

Grants, L., Powell, B., Gessel, C., Hiser, F., and Hassen, A. (2017). Gait Deficits under dual - task conditions in the concussed adolescent and young athlete population: a systematic review. Int. J. Sports Phys. Ther. 12, 1011-1022. doi: 10.26603/ijspt20171011

Guedes, V. A., Kenney, K., Shahim, P., Qu, B.-X., Lai, C., Devoto, C., et al. (2020). Exosomal neurofilament light. Neurology 94:e2412. doi: 10.1212/WNL. 0000000000009577

Gupta, R., and Sen, N. (2016). Traumatic brain injury: a risk factor for neurodegenerative diseases. Rev. Neurosci. 27, 93-100. doi: 10.1515/revneuro2015-0017

Guskiewicz, K. M., Marshall, S. W., Bailes, J., McCrea, M., Cantu, R. C., Randolph, C., et al. (2005). Association between recurrent concussion and late-life cognitive impairment in retired professional football players. Neurosurgery 57, 719-726. doi: 10.1093/neurosurgery/57.4.719

Hakansson, K., Ledreux, A., Daffner, K., Terjestam, Y., Bergman, P., Carlsson, R., et al. (2017). BDNF responses in healthy older persons to 35 minutes of physical exercise, cognitive training, and mindfulness: associations with working memory function. J. Alzheimers Dis. 55, 645-657. doi: 10.3233/JAD160593

Hamlett, E. D., Larosa, A., Mufson, E. J., Fortea, J., Ledreux, A., and Granholm, A. C. (2019). Exosome release and cargo in Down syndrome. Dev. Neurobiol. 79, 639-655. doi: 10.1002/dneu.22712

Haque, A., Polcyn, R., Matzelle, D., and Banik, N. (2018). New Insights into the role of neuron-specific enolase in neuroinflammation, neurodegeneration, and neuroprotection. Brain Sci 8:33. doi: 10.3390/brainsci8020033

Harmon, K. G., Drezner, J. A., Gammons, M., Guskiewicz, K. M., Halstead, M., Herring, S. A., et al. (2013). American medical society for sports medicine position statement: concussion in sport. Br. J. Sports Med. 47, 15-26. doi: 10.1136/bjsports-2012-091941

Heneka, M. T., Carson, M. J., El Khoury, J., Landreth, G. E., Brosseron, F., Feinstein, D. L., et al. (2015). Neuroinflammation in Alzheimer's disease. Lancet Neurol. 14, 388-405. doi: 10.1016/S1474-4422(15)70016-5

Higgins, K. L., Denney, R. L., and Maerlender, A. (2017). Sandbagging on the immediate post-concussion assessment and cognitive testing (ImPACT) in a high school athlete population. Arch. Clin. Neuropsychol. 32, 259-266. doi: $10.1093 / \mathrm{arclin} / \mathrm{acw} 108$
Hobbs, J., Young, J., and Bailes, J. (2016). Sports-related concussions: diagnosis, complications, and current management strategies. Neurosurg. Focus 40:E5.

Howell, D. R., Kirkwood, M. W., Provance, A., Iverson, G. L., and Meehan, W. P. (2018a). Using concurrent gait and cognitive assessments to identify impairments after concussion: a narrative review. Concussion 3:CNC54.

Howell, D. R., Stillman, A., Buckley, T. A., Berkstresser, B., Wang, F., and Meehan, W. P. (2018b). The utility of instrumented dual-task gait and tablet-based neurocognitive measurements after concussion. J. Sci. Med. Sport 21, 358-362. doi: 10.1016/j.jsams.2017.08.004

Ignowski, E., Winter, A. N., Duval, N., Fleming, H., Wallace, T., Manning, E., et al. (2018). The cysteine-rich whey protein supplement, Immunocal(R), preserves brain glutathione and improves cognitive, motor, and histopathological indices of traumatic brain injury in a mouse model of controlled cortical impact. Free Radic. Biol. Med. 124, 328-341. doi: 10.1016/j.freeradbiomed.2018.06.026

Iliff, J. J., Chen, M. J., Plog, B. A., Zeppenfeld, D. M., Soltero, M., Yang, L., et al. (2014). Impairment of glymphatic pathway function promotes tau pathology after traumatic brain injury. J. Neurosci. 34, 16180-16193. doi: 10. 1523/JNEUROSCI.3020-14.2014

Israel, I., Ohsiek, A., Al-Momani, E., Albert-Weissenberger, C., Stetter, C., Mencl, S., et al. (2016). Combined [(18)F]DPA-714 micro-positron emission tomography and autoradiography imaging of microglia activation after closed head injury in mice. J. Neuroinflamm. 13:140. doi: 10.1186/s12974-016-0604-9

Iverson, G. L., Gardner, A. J., Terry, D. P., Ponsford, J. L., Sills, A. K., Broshek, D. K., et al. (2017). Predictors of clinical recovery from concussion: a systematic review. Br. J. Sports Med. 51, 941-948. doi: 10.1136/bjsports-2017-097729

Jassam, Y., Izzy, S., Whalen, M., McGavern, D., and El Khoury, J. (2017). Neuroimmunology of traumatic brain injury: time for a paradigm shift. Neuron 95, 1246-1265. doi: 10.1016/j.neuron.2017.07.010

Jessen, N. A., Munk, A. S., Lundgaard, I., and Nedergaard, M. (2015). The glymphatic system: a beginner's guide. Neurochem. Res. 40, 2583-2599. doi: 10.1007/s11064-015-1581-6

Johansson, H., Lundin-Olsson, L., Littbrand, H., Gustafson, Y., Rosendahl, E., and Toots, A. (2017). Cognitive function and walking velocity in people with dementia; a comparison of backward and forward walking. Gait Posture 58, 481-486. doi: 10.1016/j.gaitpost.2017.09.009

Johnson, V. E., Stewart, W., Arena, J. D., and Smith, D. H. (2017). Traumatic brain injury as a trigger of neurodegeneration. Adv. Neurobiol. 15, 383-400. doi: 10.1007/978-3-319-57193-5_15

Johnson, V. E., Stewart, W., Weber, M. T., Cullen, D. K., Siman, R., and Smith, D. H. (2016). SNTF immunostaining reveals previously undetected axonal pathology in traumatic brain injury. Acta Neuropathol. 131, 115-135. doi: 10. 1007/s00401-015-1506-0

Joseph, J. R., Swallow, J. S., Willsey, K., Lapointe, A. P., Khalatbari, S., Korley, F. K., et al. (2018). Elevated markers of brain injury as a result of clinically asymptomatic high-acceleration head impacts in high-school football athletes. J. Neurosurg. 3, 1-7. doi: 10.3171/2017.12.JNS172386

Kaijzel, E., Van Beek, E., Stammes, M., Que, I., Chan, A., Lowik, C., et al. (2017). Traumatic brain injury: preclinical imaging diagnostic(s) and therapeutic approaches. Curr. Pharm. Des 23, 1909-1915. doi: 10.2174/ 1381612823666170116141116

Kalajainen, A. (2015). Comparison of the Balance Error Scoring System and the NeuroCom Sensory Organization Test in healthy, physically active adults. Doctoral dissertation. Pittsburgh: University of Pittsburgh.

Kalani, A., Tyagi, A., and Tyagi, N. (2014). Exosomes: mediators of neurodegeneration, neuroprotection and therapeutics. Mol. Neurobiol. 49, 590-600. doi: 10.1007/s12035-013-8544- 1

Kane, M. J., Angoa-Perez, M., Briggs, D. I., Viano, D. C., Kreipke, C. W., and Kuhn, D. M. (2012). A mouse model of human repetitive mild traumatic brain injury. J. Neurosci. Methods 203, 41-49. doi: 10.1016/j.jneumeth.2011.09.003

Kane, R. L., Roebuck-Spencer, T., Short, P., Kabat, M., and Wilken, J. (2007). Identifying and monitoring cognitive deficits in clinical populations using Automated Neuropsychological Assessment Metrics (ANAM) tests. Arch. Clin. Neuropsychol. 22(Suppl. 1), S115-S126. doi: 10.1016/j.acn.2006.10.006

Kaplan, G., Vasterling, J., and Vedak, P. (2010). Brain-derived neurotrophic factor in traumatic brain injury, post-traumatic stress disorder, and their comorbid conditions: role in pathogenesis and treatment. Behav. Pharmacol. 21, 427-437.

Kapogiannis, D., Mustapic, M., Shardell, M. D., Berkowitz, S. T., Diehl, T. C., Spangler, R. D., et al. (2019). Association of extracellular vesicle biomarkers with 
alzheimer disease in the baltimore longitudinal study of aging. JAMA Neurol. 76, 1340-1351. doi: 10.1001/jamaneurol.2019.2462

Kasamatsu, T., Cleary, M., Bennett, J., Howard, K., and McLeod, T. V. (2016). Examining academic support after concussion for the adolescent studentathlete: perspectives of the athletic trainer. J. Athl. Train 51, 153-161. doi: 10.4085/1062-6050-51.4.02

Kaufman, S. K., Sanders, D. W., Thomas, T. L., Ruchinskas, A. J., Vaquer-Alicea, J., Sharma, A. M., et al. (2016). Tau prion strains dictate patterns of cell pathology, progression rate, and regional vulnerability in vivo. Neuron 92, 796-812. doi: 10.1016/j.neuron.2016.09.055

Kawata, K., Liu, C. Y., Merkel, S. F., Ramirez, S. H., Tierney, R. T., and Langford, D. (2016). Blood biomarkers for brain injury: what are we measuring? Neurosci. Biobehav. Rev. 68, 460-473. doi: 10.1016/j.neubiorev.2016.05.009

Kenney, K., Qu, B. X., Lai, C., Devoto, C., Motamedi, V., Walker, W. C., et al. (2018). Higher exosomal phosphorylated tau and total tau among veterans with combat-related repetitive chronic mild traumatic brain injury. Brain Inj. 32, 1276-1284. doi: 10.1080/02699052.2018.1483530

King, D., Gissane, C., Hume, P. A., and Flaws, M. (2015). The King-Devick test was useful in management of concussion in amateur rugby union and rugby league in New Zealand. J. Neurol. Sci. 351, 58-64. doi: 10.1016/j.jns.2015.02.035

Knapstad, M. K., Steihaug, O. M., Aaslund, M. K., Nakling, A., Naterstad, I. F., Fladby, T., et al. (2019). Reduced walking speed in subjective and mild cognitive impairment: a cross-sectional study. J. Geriatr. Phys. Ther. 42, E122-E128. doi: 10.1519/JPT.0000000000000157

Ko, J., Hemphill, M., Yang, Z., Sewell, E., Na, Y., Sandsmark, D., et al. (2018). Diagnosis of traumatic brain injury using miRNA signatures in nanomagnetically isolated brain-derived extracellular vesicles. Lab Chip 18, 3617-3630. doi: 10.1039/c81c00672e

Kontos, A. P., Braithwaite, R., Dakan, S., and Elbin, R. J. (2014). Computerized neurocognitive testing within 1 week of sport-related concussion: meta-analytic review and analysis of moderating factors. J. Int. Neuropsychol. Soc. 20, 324-332. doi: $10.1017 /$ S1355617713001471

Korley, F. K., Diaz-Arrastia, R., Wu, A. H., Yue, J. K., Manley, G. T., Sair, H. I., et al. (2016). Circulating brain-derived neurotrophic factor has diagnostic and prognostic value in traumatic brain injury. J. Neurotrauma 33, 215-225. doi: $10.1089 /$ neu. 2015.3949

Kou, Z., Gattu, R., Kobeissy, F., Welch, R., O’neil, B., Woodard, J., et al. (2013). Combining biochemical and imaging markers to improve diagnosis and characterization of mild traumatic brain injury in the acute setting: results from a pilot study. PLoS ONE 8:e80296. doi: 10.1371/journal.pone.0080296

Kroshus, E., Garnett, B., Hawrilenko, M., Baugh, C. M., and Calzo, J. P. (2015). Concussion under-reporting and pressure from coaches, teammates, fans, and parents. Soc. Sci. Med. 134, 66-75. doi: 10.1016/j.socscimed.2015.04.011

Kulbe, J. R., and Geddes, J. W. (2016). Current status of fluid biomarkers in mild traumatic brain injury. Exp. Neurol. 275(Pt 3), 334-352. doi: 10.1016/j. expneurol.2015.05.004

Kutcher, J. S., and Giza, C. C. (2014). Sports concussion diagnosis and management. Continuum (Minneap Minn) 20, 1552-1569. doi: 10.1212/01. CON.0000458974.78766.58

Laurer, H. L., Bareyre, F. M., Lee, V. M., Trojanowski, J. Q., Longhi, L., and Hoover, R. (2001). Mild head injury increasing the brain's vulnerability to a second concussive impact. J. Neurosurg. 95, 859-870. doi: 10.3171/jns.2001.95.5.0859

Leclerc, S., Lassonde, M., Delaney, J. S., Lacroix, V. J., and Johnston, K. M. (2001). Recommendations for grading of concussion in athletes. Sports Med. 31, 629-636. doi: 10.2165/00007256-200131080-00007

Leddy, J. J., Baker, J. G., and Willer, B. (2016). Active rehabilitation of concussion and post-concussion syndrome. Phys. Med. Rehabil. Clin. N. Am. 27, 437-454. doi: 10.1016/j.pmr.2015.12.003

Ledreux, A., Håkansson, K., Carlsson, R., Kidane, M., Columbo, L., and Terjestam, Y. (2019). Differential effects of physical exercise, cognitive training and mindfulness practice on serum BDNF levels in healthy older adults: a randomized controlled unsupervised intervention study. J. Alzheimers Dis. 71, 1245-1261. doi: 10.3233/JAD- 190756

Lepage, C., De Pierrefeu, A., Koerte, I. K., Coleman, M. J., Pasternak, O., Grant, G., et al. (2018). White matter abnormalities in mild traumatic brain injury with and without post-traumatic stress disorder: a subject-specific diffusion tensor imaging study. Brain Imaging Behav. 12, 870-881. doi: 10.1007/s11682-0179744-5
Li, Y., Yang, Y., Ren, J., Xu, F., Chen, F., and Li, A. (2017). Exosomes secreted by stem cells from human exfoliated deciduous teeth contribute to functional recovery after traumatic brain injury by shifting microglia M1/M2 polarization in rats. Stem Cell Res. Ther. 8:198. doi: 10.1186/s13287-017-0648-5

Liaw, M. Y., Chen, C. L., Pei, Y. C., Leong, C. P., and Lau, Y. C. (2009). Comparison of the static and dynamic balance performance in young, middle-aged, and elderly healthy people. Change Gung. Med. J 32, 297-304.

Ling, H., Hardy, J., and Zetterberg, H. (2015). Neurological consequences of traumatic brain injuries in sports. Mol. Cell. Neurosci. 66, 114-122. doi: 10.1016/ j.mcn.2015.03.012

Liu, C., Chen, S., Dietrich, D., and Hu, B. (2008). Changes in autophagy after traumatic brain injury. J. Cereb. Blood Flow Metab. 28, 675-683. doi: 10.1038/sj. jcbfm. 9600587

Lobue, C., Schaffert, J., Cullum, C. M., Peters, M. E., Didehbani, N., Hart, J., et al. (2020). Clinical and neuropsychological profile of patients with dementia and chronic traumatic encephalopathy. J. Neurol. Neurosurg. Psychiatry 91:586. doi: 10.1136/jnnp-2019-321567

Luo, J., Nguyen, A., Villeda, S., Zhang, H., Ding, Z., Lindsey, D., et al. (2014). Longterm cognitive impairments and pathological alterations in a mouse model of repetitive mild traumatic brain injury. Front. Neurol. 5:12. doi: 10.3389/fneur. 2014.00012

Lyeth, B. G. (2016). Historical review of the fluid-percussion TBI model. Front. Neurol. 7:217. doi: 10.3389/fneur.2016.00217

Majerske, C. W., Mihalik, J. P., Ren, D., Collins, M. W., Reddy, C. C., Lovell, M. R., et al. (2008). Concussion in sports: postconcussive activity levels, symptoms, and neurocognitive performance. J. Athl. Train 43, 265-274. doi: 10.4085/10626050-43.3.265

Manek, R., Moghieb, A., Yang, Z., Kumar, D., Kobessiy, F., Ga, S., et al. (2018). Protein biomarkers and neuroproteomics characterization of microvesicles/exosomes from human cerebrospinal fluid following traumatic brain injury. Mol. Neurobiol. 55, 6112-6128. doi: 10.1007/s12035-017-0821-y

Manley, G., Gardner, A. J., Schneider, K. J., Guskiewicz, K. M., Bailes, J., Cantu, R. C., et al. (2017). A systematic review of potential long-term effects of sportrelated concussion. Br. J. Sports Med. 51, 969-977. doi: 10.1136/bjsports-2017097791

Mannix, R., Meehan, W. P., Mandeville, J., Grant, P. E., Gray, T., Berglass, J., et al. (2013). Clinical correlates in an experimental model of repetitive mild brain injury. Ann. Neurol. 74, 65-75. doi: 10.1002/ana.23858

Martini, D. N., Eckner, J. T., Meehan, S. K., and Broglio, S. P. (2017). Long-term effects of adolescent sport concussion across the age spectrum. Am. J. Sports Med. 45, 1420-1428. doi: 10.1177/0363546516686785

McAllister, T., and McCrea, M. (2017). Long-term cognitive and neuropsychiatric consequences of repetitive concussion and head-impact exposure. J Athl Train 52, 309-317. doi: 10.4085/1062-6050-52.1.14

McAteer, K. M., Corrigan, F., Thornton, E., Turner, R. J., and Vink, R. (2016). Short and long term behavioral and pathological changes in a novel rodent model of repetitive mild traumatic brain injury. PLoS ONE 11:e0160220. doi: 10.1371/journal.pone. 0160220

McCrea, M., Broglio, S. P., McAllister, T. W., Gill, J., Giza, C. C., Huber, D. L., et al. (2020). Association of blood biomarkers with acute sport-related concussion in collegiate athletes: findings from the NCAA and department of defense CARE consortium. JAMA Netw Open 3:e1919771. doi: 10.1001/jamanetworkopen. 2019.19771

McCrory, P., Feddermann-Demont, N., Dvorak, J., Cassidy, J. D., McIntosh, A., Vos, P. E., et al. (2017). What is the definition of sports-related concussion: a systematic review. Br. J. Sports Med. 51, 877-887. doi: 10.1136/bjsports-2016097393

McDonald, T., Burghart, M. A., and Nazir, N. (2016). Underreporting of concussions and concussion-like symptoms in female high school athletes. $J$ Trauma Nurs. 23, 241-246. doi: 10.1097/JTN.0000000000000227

McIinnes, K., Friesen, C. L., Mackenzie, D. E., Westwood, D. A., and Boe, S. G. (2017). Mild traumatic brain injury (mTBI) and chronic cognitive impairment: a scoping review. PLoS ONE 12:e0174847. doi: 10.1371/journal.pone.0174847

McKee, A. C., Cairns, N. J., Dickson, D. W., Folkerth, R. D., Keene, C. D., Litvan, I., et al. (2016). The first NINDS/NIBIB consensus meeting to define neuropathological criteria for the diagnosis of chronic traumatic encephalopathy. Acta Neuropathol. 131, 75-86. doi: 10.1007/s00401-015$1515-z$ 
McKee, A. C., and Daneshvar, D. H. (2015). The neuropathology of traumatic brain injury. Handb. Clin. Neurol. 127, 45-66. doi: 10.1016/B978-0-444-528926.00004-0

McLeod, T. C., and Leach, C. (2012). Psychometric properties of self-report concussion scales and checklists. J. Athl. Train 47, 221-223. doi: 10.4085/10626050-47.2.221

Meier, T., Nelson, L., Huber, D., Bazarian, J., Hayes, R., and McCrea, M. (2017). Prospective assessment of acute blood markers of brain injury in sport-related concussion. J. Neurotrauma 34, 3134-3142. doi: 10.1089/neu.2017.5046

Mercier, E., Tardif, P., Cameron, P., Émond, M., Moore, L., and Mitra, B. (2018). Prognostic value of neuron-specific enolase (NSE) for prediction of postconcussion symptoms following a mild traumatic brain injury: a systematic review. Brain Inj. 32, 29-40. doi: 10.1080/02699052.2017.1385097

Mez, J., Daneshvar, D. H., Kiernan, P. T., Abdolmohammadi, B., Alvarez, V. E., Huber, B. R., et al. (2017). Clinicopathological evaluation of chronic traumatic encephalopathy in players of american football. JAMA 318, 360-370. doi: 10 . 1001/jama.2017.8334

Miyashita, T. L., Diakogeorgiou, E., and Marrie, K. (2017). Correlation of head impacts to change in balance error scoring system scores in division i men's lacrosse players. Sports Health 9, 318-323. doi: 10.1177/1941738116685306

Mondello, S., Thelin, E. P., Shaw, G., Salzet, M., Visalli, C., Cizkova, D., et al. (2018). Extracellular vesicles: pathogenetic, diagnostic and therapeutic value in traumatic brain injury. Expert Rev. Proteom. 15, 451-461. doi: 10.1080/ 14789450.2018.1464914

Mouzon, B., Chaytow, H., Crynen, G., Bachmeier, C., Stewart, J., Mullan, M., et al. (2012). Repetitive mild traumatic brain injury in a mouse model produces learning and memory deficits accompanied by histological changes. J. Neurotrauma 29, 2761-2773. doi: 10.1089/neu.2012.2498

Mouzon, B. C., Bachmeier, C., Ferro, A., Ojo, J. O., Crynen, G., Acker, C. M., et al. (2014). Chronic neuropathological and neurobehavioral changes in a repetitive mild traumatic brain injury model. Ann. Neurol. 75, 241-254. doi: 10.1002/ana.24064

Mouzon, B. C., Bachmeier, C., Ojo, J. O., Acker, C. M., Ferguson, S., Paris, D., et al. (2018). Lifelong behavioral and neuropathological consequences of repetitive mild traumatic brain injury. Ann. Clin. Transl. Neurol. 5, 64-80. doi: 10.1002/ acn 3.510

Mulligan, I., Boland, M., and McIlhenny, C. (2013). The balance error scoring system learned response among young adults. Sports Health 5, 22-26. doi: $10.1177 / 1941738112467755$

Neselius, S., Brisby, H., Theodorsson, A., Blennow, K., Zetterberg, H., and Marcusson, J. (2012). CSF-biomarkers in Olympic boxing: diagnosis and effects of repetitive head trauma. PLOS ONE 7:e33606. doi: 10.1371/journal.pone. 0033606

Nitta, M. E., Savitz, J., Nelson, L. D., Teague, T. K., Hoelzle, J. B., McCrea, M. A., et al. (2019). Acute elevation of serum inflammatory markers predicts symptom recovery after concussion. Neurology 93, e497-e507. doi: 10.1212/ WNL.0000000000007864

O'connell, B., Kelly, A. M., Mockler, D., Oresic, M., Denvir, K., Farrell, G., et al. (2018). Use of blood biomarkers in the assessment of sports-related concussiona systematic review in the context of their biological significance. Clin. J. Sport. Med. 28, 561-571. doi: 10.1097/JSM.0000000000000478

O'connor, K. L., Baker, M. M., Dalton, S. L., Dompier, T. P., Broglio, S. P., and Kerr, Z. Y. (2017). Epidemiology of sport-related concussions in high school athletes: national athletic treatment, injury and outcomes network (NATION), 2011-2012 through 2013-2014. J. Athl. Train 52, 175-185. doi: 10.4085/10626050-52.1.15

Ojo, J. O., Mouzon, B. C., and Crawford, F. (2016). Repetitive head trauma, chronic traumatic encephalopathy and tau: challenges in translating from mice to men. Exp. Neurol. 275(Pt 3), 389-404. doi: 10.1016/j.expneurol.2015.06.003

O’keeffe, E., Kelly, E., Liu, Y., Giordano, C., Wallace, E., Hynes, M., et al. (2019). Dynamic blood-brain barrier regulation in mild traumatic brain injury. J. Neurotrauma 37, 347-356. doi: 10.1089/neu.2019.6483

Olczak, M., Niderla-Bielinska, J., Kwiatkowska, M., Samojlowicz, D., Tarka, S., and Wierzba-Bobrowicz, T. (2017). Tau protein (MAPT) as a possible biochemical marker of traumatic brain injury in postmortem examination. Foren. Sci. Int. 280, 1-7. doi: 10.1016/j.forsciint.2017.09.008

Omalu, B. (2014). Chronic traumatic encephalopathy. Prog. Neurol. Surg. 28, 38-49. doi: $10.1159 / 000358761$
Osier, N., and Dixon, C. E. (2016). The controlled cortical impact model of experimental brain trauma: overview, research applications, and protocol. Methods Mol. Biol. 1462, 177-192. doi: 10.1007/978-1-4939-3816-2_11

Papa, L. (2014). Biomarkers for concussion. 235-248. doi: 10.1007/978-1-49390295-8 13

Papa, L., Ramia, M. M., Edwards, D., Johnson, B. D., and Slobounov, S. M. (2015). Systematic review of clinical studies examining biomarkers of brain injury in athletes after sports-related concussion. J. Neurotrauma 32, 661-673. doi: 10.1089/neu.2014.3655

Patricios, J. S., Ardern, C. L., Hislop, M. D., Aubry, M., Bloomfield, P., Broderick, C., et al. (2018). Implementation of the 2017 berlin concussion in sport group consensus statement in contact and collision sports: a joint position statement from 11 national and international sports organisations. Br. J. Sports Med. 52, 635-641. doi: 10.1136/bjsports-2018-099079

Patterson, Z., and Holahan, M. (2012). Understanding the neuroinflammatory response following concussion to develop treatment strategies. Front. Cell Neurosci. 6:58. doi: 10.3389/fncel.2012.00058

Petraglia, A. L., Plog, B. A., Dayawansa, S., Dashnaw, M. L., Czerniecka, K., Walker, C. T., et al. (2014). The pathophysiology underlying repetitive mild traumatic brain injury in a novel mouse model of chronic traumatic encephalopathy. Surg. Neurol. Int. 5:184. doi: 10.4103/2152-7806.147566

Plog, B. A., Dashnaw, M. L., Hitomi, E., Peng, W., Liao, Y., Lou, N., et al. (2015). Biomarkers of traumatic injury are transported from brain to blood via the glymphatic system. J. Neurosci. 35:518. doi: 10.1523/JNEUROSCI.3742-14.2015

Plog, B. A., and Nedergaard, M. (2015). Why have we not yet developed a simple blood test for TBI? Exp. Rev. Neurotherapeut. 15, 465-468. doi: 10.1586/ 14737175.2015 .1031112

Powers, K. C., Kalmar, J. M., and Cinelli, M. E. (2014). Dynamic stability and steering control following a sport-induced concussion. Gait Posture 39, 728732. doi: 10.1016/j.gaitpost.2013.10.005

Prins, M. L., Hales, A., Reger, M., Giza, C. C., and Hovda, D. A. (2010). Repeat traumatic brain injury in the juvenile rat is associated with increased axonal injury and cognitive impairments. Dev. Neurosci. 32, 510-518. doi: 10.1159/ 000316800

Raj, A., Kuceyeski, A., and Weiner, M. (2012). A network diffusion model of disease progression in dementia. Neuron 73, 1204-1215. doi: 10.1016/j.neuron.2011.12. 040

Ramont, L., Thoannes, H., Volondat, A., Chastang, F., Millet, M. C., and Maquart, F. X. (2005). Effects of hemolysis and storage condition on neuron-specific enolase (NSE) in cerebrospinal fluid and serum: implications in clinical practice. Clin. Chem. Lab. Med. 43, 1215-1217. doi: 10.1515/CCLM.2005.210

Reynolds, B. B., Patrie, J., Henry, E. J., Goodkin, H. P., Broshek, D. K., Wintermark, M., et al. (2016). Quantifying head impacts in collegiate lacrosse. Am. J. Sports Med. 44, 2947-2956. doi: 10.1177/0363546516648442

Rodriguez-Grande, B., Obenaus, A., Ichkova, A., Aussudre, J., Bessy, T., Barse, E., et al. (2018). Gliovascular changes precede white matter damage and longterm disorders in juvenile mild closed head injury. Glia 66, 1663-1677. doi: $10.1002 /$ glia.23336

Sajja, V., Hlavac, N., and Vandevord, P. (2016). Role of glia in memory deficits following traumatic brain injury: biomarkers of glia dysfunction. Front. Integr. Neurosci. 10:7. doi: 10.3389/fnint.2016.00007

Schatz, P., Pardini, J. E., Lovell, M. R., Collins, M. W., and Podell, K. (2006). Sensitivity and specificity of the ImPACT Test battery for concussion in athletes. Arch. Clin. Neuropsychol. 21, 91-99. doi: 10.1016/j.acn.2005.08.001

Scott, B. R., Uomoto, J. M., and Barry, E. S. (2020). Impact of pre-existing migraine and other co-morbid or co-occurring conditions on presentation and clinical course following deployment-related concussion. Headache 60, 526-541. doi: 10.1111/head.13709

Setsuie, R., and Wada, K. (2007). The functions of UCH-L1 and its relation to neurodegenerative diseases. Neurochem. Int. 51, 105-111. doi: 10.1016/j.neuint. 2007.05.007

Shahim, P., Tegner, Y., Marklund, N., Hoglund, K., Portelius, E., Brody, D. L., et al. (2017). Astroglial activation and altered amyloid metabolism in human repetitive concussion. Neurology 88, 1400-1407. doi: 10.1212/WNL. 0000000000003816

Shahjouei, S., Sadeghi-Naini, M., Yang, Z., Kobeissy, F., Rathore, D., Shokraneh, F., et al. (2018). The diagnostic values of UCH-L1 in traumatic brain injury: a meta-analysis. Brain Inj. 32, 1-17. doi: 10.1080/02699052.2017.1382717 
Shitaka, Y., Tran, H. T., Bennett, R. E., Sanchez, L., Levy, M. A., Dikranian, K., et al. (2011). Repetitive closed-skull traumatic brain injury in mice causes persistent multifocal axonal injury and microglial reactivity. J. Neuropathol. Exp. Neurol. 70, 551-567. doi: 10.1097/NEN.0b013e31821f891f

Siman, R., Shahim, P., Tegner, Y., Blennow, K., Zetterberg, H., and Smith, D. H. (2015). Serum SNTF increases in concussed professional ice hockey players and relates to the severity of postconcussion symptoms. J. Neurotrauma 32, 1294-1300. doi: 10.1089/neu.2014.3698

Simmons, M. M., Swedler, D. I., and Kerr, Z. Y. (2017). Injury surveillance of head, neck, and facial injuries in collegiate ice hockey players, 2009-2010 through 2013-2014 academic years. J. Athl. Train 52, 776-784. doi: 10.4085/1062-605052.4 .03

Singh, K., Trivedi, R., Devi, M., Tripathi, R., and Khushu, S. (2016). Longitudinal changes in the DTI measures, anti-GFAP expression and levels of serum inflammatory cytokines following mild traumatic brain injury. Exp. Neurol. 275, 427-435. doi: 10.1016/j.expneurol.2015.07.016

Smith, C., Chen, Y., Sullivan, M., Kochanek, P., and Clark, R. (2011). Autophagy in acute brain injury: feast, famine, or folly? Neurobiol. Dis. 43, 52-59. doi: 10.1016/j.nbd.2010.09.014

Sosnoff, J. J., Broglio, S. P., Shin, S., and Ferrara, M. S. (2011). Previous mild traumatic brain injury and postural-control dynamics. J. Athl. Train 46, 85-91. doi: 10.4085/1062-6050-46.1.85

Stalnacke, B. M., Tegner, Y., and Sojka, P. (2004). Playing soccer increases serum concentrations of the biochemical markers of brain damage S-100B and neuron-specific enolase in elite players: a pilot study. Brain Inj. 18, 899-909. doi: 10.1080/02699050410001671865

Stern, R. A., Tripodis, Y., Baugh, C. M., Fritts, N. G., Martin, B. M., Chaisson, C., et al. (2016). Preliminary study of plasma exosomal tau as a potential biomarker for chronic traumatic encephalopathy. J. Alzheimers. Dis. 51, 1099-1109. doi: 10.3233/JAD- 151028

Sullan, M. J., Asken, B. M., Jaffee, M. S., Dekosky, S. T., and Bauer, R. M. (2018). Glymphatic system disruption as a mediator of brain trauma and chronic traumatic encephalopathy. Neurosci. Biobehav. Rev. 84, 316-324. doi: 10.1016/ j.neubiorev.2017.08.016

Susarla, B. T., Villapol, S., Yi, J. H., Geller, H. M., and Symes, A. J. (2014). Temporal patterns of cortical proliferation of glial cell populations after traumatic brain injury in mice. ASN Neuro 6, 159-170. doi: 10.1042/AN20130034

Tagge, C. A., Fisher, A. M., Minaeva, O. V., Gaudreau-Balderrama, A., Moncaster, J. A., Zhang, X. L., et al. (2018). Concussion, microvascular injury, and early tauopathy in young athletes after impact head injury and an impact concussion mouse model. Brain 141, 422-458. doi: 10.1093/brain/awx350

Tajiri, N., Kellogg, S. L., Shimizu, T., Arendash, G. W., and Borlongan, C. V. (2013). Traumatic brain injury precipitates cognitive impairment and extracellular Abeta aggregation in Alzheimer's disease transgenic mice. PLoS ONE 8:e78851. doi: 10.1371/journal.pone.0078851

Thomas, D. G., Collins, M. W., Saladino, R. A., Frank, V., Raab, J., and Zuckerbraun, N. S. (2011). Identifying neurocognitive deficits in adolescents following concussion. Acad. Emerg. Med. 18, 246-254. doi: 10.1111/j.15532712.2011.01015.x

Tracy, T. E., and Gan, L. (2018). Tau-mediated synaptic and neuronal dysfunction in neurodegenerative disease. Curr. Opin. Neurobiol. 51, 134-138. doi: 10.1016/ j.conb.2018.04.027

Turner, R. C., Lucke-Wold, B. P., Robson, M. J., Lee, J. M., and Bailes, J. E. (2016). Alzheimer's disease and chronic traumatic encephalopathy: distinct but possibly overlapping disease entities. Brain Inj. 30, 1279-1292. doi: 10.1080/02699052. 2016.1193631

Uryu, K., Laurer, H., McIntosh, T., Pratico, D., Martinez, D., Leight, S., et al. (2002). Repetitive mild brain trauma accelerates Abeta deposition, lipid peroxidation, and cognitive impairment in a transgenic mouse model of Alzheimer amyloidosis. J. Neurosci. 22, 446-454. doi: 10.1523/jneurosci.22-0200446.2002

Valovich, T. C., Perrin, D. H., Guskiewicz, K. M., Shultz, S. J., Diamond, R., and Gansneder, B. M. (2004). Serial administration of clinical concussion assessments and learning effects in healthy young athletes. Clin. J. Sport. Med 14, 287-295. doi: 10.1097/00042752-200409000-00007

Voormolen, D. C., Polinder, S., Von Steinbuechel, N., Vos, P. E., Cnossen, M. C., and Haagsma, J. A. (2019). The association between post-concussion symptoms and health-related quality of life in patients with mild traumatic brain injury. Inj. Int. J. Care Inj. 50, 1068-1074. doi: 10.1016/j.injury.2018.12.002

Wallace, C., Zetterberg, H., Blennow, K., and Van Donkelaar, P. (2018). No change in plasma tau and serum neurofilament light concentrations in adolescent athletes following sport-related concussion. PLoS ONE 13:e0206466. doi: 10. 1371/journal.pone.0206466

Wallace, J., Covassin, T., Nogle, S., Gould, D., and Kovan, J. (2017). Concussion knowledge and reporting behavior differences between high school athletes at urban and suburban high schools. J. Sch. Health 87, 665-674. doi: 10.1111/josh. 12543

Washington, P., Villapol, S., and Burns, M. (2016). Polypathology and dementia after brain trauma: does brain injury trigger distinct neurodegenerative diseases, or should they be classified together as traumatic encephalopathy? Exp. Neurol. 275, 381-388. doi: 10.1016/j.expneurol.2015.06.015

Weston, W., Ganey, T., and Temple, H. (2019). The relationship between exosomes and cancer: implications for diagnostics and therapeutics. BioDrugs 33, 137158. doi: 10.1007/s40259-019-00338-5

Winston, C. N., Aulston, B., Rockenstein, E. M., Adame, A., Prikhodko, O., Dave, K. N., et al. (2019a). Neuronal exosome-derived human tau is toxic to recipient mouse neurons in vivo. J. Alzheimers Dis. 67, 541-553. doi: 10.3233/JAD180776

Winston, C. N., Romero, H. K., Ellisman, M., Nauss, S., Julovich, D. A., Conger, T., et al. (2019b). Assessing neuronal and astrocyte derived exosomes from individuals with mild traumatic brain injury for markers of neurodegeneration and cytotoxic activity. Front. Neurosci. 13:1005. doi: 10.3389/fnins.2019.01005

Winston, C. N., Noel, A., Neustadtl, A., Parsadanian, M., Barton, D. J., Chellappa, D., et al. (2016). Dendritic spine loss and chronic white matter inflammation in a mouse model of highly repetitive head trauma. Am. J. Pathol. 186, 552-567. doi: 10.1016/j.ajpath.2015.11.006

Wojnarowicz, M. W., Fisher, A. M., Minaeva, O., and Goldstein, L. E. (2017). Considerations for experimental animal models of concussion, traumatic brain injury, and chronic traumatic encephalopathy-these matters matter. Front. Neurol. 8:240. doi: 10.3389/fneur.2017.00240

Xiong, Y., Mahmood, A., and Chopp, M. (2017a). Animal models of traumatic brain injuries. Nat. Rev. Neurosci. 14, 128-142. doi: 10.1038/nrn3407

Xiong, Y., Mahmood, A., and Chopp, M. (2017b). Emerging potential of exosomes for treatment of traumatic brain injury. Neural Regen. Res. 12, 19-22. doi: 10.4103/1673-5374.198966

Yang, Y., Ye, Y., Su, X., He, J., Bai, W., and He, X. (2017). MSCs-derived exosomes and neuroinflammation, neurogenesis and therapy of traumatic brain injury. Front. Cell Neurosci. 11:55. doi: 10.3389/fncel.2017.00055

Yang, Z., Wang, P., Morgan, D., Lin, D., Pan, J., Lin, F., et al. (2015). Temporal MRI characterization, neurobiochemical and neurobehavioral changes in a mouse repetitive concussive head injury model. Sci. Rep. 5:11178. doi: 10.1038/ srep 11178

Yates, N. J., Lydiard, S., Fehily, B., Weir, G., Chin, A., Bartlett, C. A., et al. (2017). Repeated mild traumatic brain injury in female rats increases lipid peroxidation in neurons. Exp. Brain Res. 235, 2133-2149. doi: 10.1007/s00221-017-4958-8

Yengo-Kahn, A. M., Hale, A. T., Zalneraitis, B. H., Zuckerman, S. L., Sills, A. K., and Solomon, G. S. (2016). The sport concussion assessment tool: a systematic review. Neurosurg. Focus 40:E6. doi: 10.3171/2016.1.FOCUS15611

Yin, B., Li, D. D., Huang, H., Gu, C. H., Bai, G. H., Hu, L. X., et al. (2019). Longitudinal changes in diffusion tensor imaging following mild traumatic brain injury and correlation with outcome. Front. Neural Circuits 13:28. doi: 10.3389/fncir.2019.00028

Yokobori, S., Hosein, K., Burks, S., Sharma, I., Gajavelli, S., and Bullock, R. (2013). Biomarkers for the clinical differential diagnosis in traumatic brain injury-a systematic review. CNS Neurosci. Ther. 19, 556-565. doi: 10.1111/cns. 12127

Yoshiyama, Y., Uryu, K., Higuchi, M., Longhi, L., Hoover, R., Fujimoto, S., et al. (2005). Enhanced neurofibrillary tangle formation, cerebral atrophy, and cognitive deficits induced by repetitive mild brain injury in a transgenic tauopathy mouse model. J. Neurotrauma 22, 1134-1141. doi: 10.1089/neu.2005. 22.1134

Zetterberg, H., Smith, D. H., and Blennow, K. (2013). Biomarkers of mild traumatic brain injury in cerebrospinal fluid and blood. Nat. Rev. Neurol. 9, 201-210. doi: $10.1038 /$ nrneurol.2013.9 
Zetterberg, H., Tanriverdi, F., Unluhizarci, K., Selcuklu, A., Kelestimur, F., and Blennow, K. (2009). Sustained release of neuron-specific enolase to serum in amateur boxers. Brain Inj. 23, 723-726. doi: 10.1080/026990509031 20399

Zhang, J., Li, S., Li, L., Li, M., Guo, C. J. Y., and Mi, S. (2015). Exosome and exosomal MicroRNA: trafficking, sorting, and function. Genom. Proteom. Bioinform. 13, 17-24. doi: 10.1016/j.gpb.2015.02.001

Zhang, L., and Wang, H. (2018). Autophagy in traumatic brain injury: a new target for therapeutic intervention. Front. Mol. Neurosci. 11:190. doi: 10.3389/fnmol. 2018.00190

Zhang, L., Wang, H., Fan, Y., Gao, Y., Li, X., Hu, Z., et al. (2017). Fucoxanthin provides neuroprotection in models of traumatic brain injury via the Nrf2-ARE and Nrf2-autophagy pathways. Sci. Rep. 7:46763.

Zhang, Y., Li, S., Chen, X., Zhang, Y., Liu, R., and Tao, L. (2008). Autophagy is activated and might protect neurons from degeneration after traumatic brain injury. Neurosci0 Bull. 24, 143-149. doi: 10.1007/s12264-008-1108-0

Zhang, Z., Buller, B., and Chopp, M. (2019). Exosomes - beyond stem cells for restorative therapy in stroke and neurological injury. Nat. Rev. Neurol. 15, 193-203. doi: 10.1038/s41582-018-0126-4
Zhou, G., and Brodsky, J. R. (2015). Objective vestibular testing of children with dizziness and balance complaints following sports-related concussions. Otolaryngol. Head Neck Surg. 152, 1133-1139. doi: 10.1177/0194599815576720

Zhu, M., Huang, Z., Ma, C., and Li, Y. (2017). An objective balance error scoring system for sideline concussion evaluation using duplex kinect sensors. Sensors (Basel) 17:2398. doi: 10.3390/s17102398

Conflict of Interest: The authors declare that the research was conducted in the absence of any commercial or financial relationships that could be construed as a potential conflict of interest.

Copyright (c) 2020 Ledreux, Pryhoda, Gorgens, Shelburne, Gilmore, Linseman, Fleming, Koza, Campbell, Wolff, Kelly, Margittai, Davidson and Granholm. This is an open-access article distributed under the terms of the Creative Commons Attribution License (CC BY). The use, distribution or reproduction in other forums is permitted, provided the original author(s) and the copyright owner(s) are credited and that the original publication in this journal is cited, in accordance with accepted academic practice. No use, distribution or reproduction is permitted which does not comply with these terms. 